\title{
THE BDS TEST AS A TEST FOR THE ADEQUACY OF A GARCH $(1,1)$ SPECIFICATION: A MONTE CARLO STUDY
}

\author{
Guglielmo Maria Caporale ${ }^{a}{ }^{*}$ Christos Ntantamis ${ }^{b}$, Theologos Pantelidis $^{b}$ \\ and Nikitas Pittis ${ }^{b}$ \\ ${ }^{a}$ Brunel University, London \\ ${ }^{b}$ University of Piraeus
}

\begin{abstract}
A bstract
In this study we examine the widely used Brock, Dechert and Scheinkman (BDS) test when applied to the logarithm of the standardized residuals of an estimated $\operatorname{GARCH}(1,1)$ model as a test for the adequacy of this specification. We review the conditions derived by De Lima (1996, E conometric Reviews, 15, 237-259) for the nuisance-parameter free property to hold, and address the issue of their necessity, using the flexible framework offered by the $\operatorname{GARCH}(1,1)$ model in terms of moment, memory and time heterogeneity properties. By means of Monte Carlo simulations, we show that the BDS test statistic still approximates the standard null distribution even for mildly explosive processes that violate the majority of the conditions. Thus, the test performs reasonably well, its empirical size being rather close to the nominal one. As a by-product of this study, we also shed light on the related issue of consistency of the QML estimators of the conditional variance parameters under various parameter configurations and alternative distributional assumptions on the innovation process.

Keywords: Nonlinearities, BDS Test, Nuisance-Parameter Free Property, Monte Carlo Analysis, GARCH(1,1) Model, QML estimator, Consistency

J EL classification: C15, C22
\end{abstract}

\footnotetext{
${ }^{*}$ We are grateful to an Associate Editor and two anonymous referees for useful comments and suggestions. Corresponding author: Professor Guglielmo Maria Caporale, Brunel Business School, Brunel University, Uxbridge, Middlesex UB8 3PH, UK. E-mail: Guglielmo-Maria.Caporale@brunel.ac.uk
} 


\section{Introduction}

The Brock, Dechert and Scheinkman (1987) test for nonlinearity (BDS test henceforth) is widely used as a misspecification test for parametric models capturing the dynamics of time series. For this purpose, it is applied to the estimated residuals from the model of interest. As these can still exhibit some form of dependence, even if the true innovations are i.i.d., the asymptotic distribution of the test statistic may be affected by the estimation procedure. This possible distortion has led researchers to consider test statistics exhibiting the so-called nuisance-parameter free property, i.e. whose asymptotic distribution is not affected by the intermediate step of parameter estimation.

Brock, Dechert and Scheinkman (1987) and Brock, Hsieh and LeBaron (1991) were the first to derive conditions for the BDS test to be nuisance-parameter free, and to carry out Monte Carlo simulations to corroborate their theoretical results. Following the work of Randles (1982), De Lima (1996) investigated further the invariance property of the BDS test, and showed that, under appropriate sufficient conditions for the series under scrutiny, the BDS test is nuisance-parameter free for linear additive models or models that can be cast into this format. This family includes the ARCH class of models, introduced by Engle (1982) and generalized by Bollerslev (1986), as long as the BDS test is applied to a modified residual series, namely the logarithm of the squared standardized residuals. Examining the "necessity" of De Lima's conditions is a difficult task analytically, and can be more conveniently achieved by means of suitably designed Monte Carlo simulations in the context of an appropriate model.

This paper investigates the "necessity" of De Lima's conditions for the BDS test statistic to have an asymptotic $\mathrm{N}(0,1)$ distribution when it is computed using the (modified) estimated standardized residuals of a GARCH $(1,1)$ model. Our aim is to analyze the properties of the BDS test when applied to the logarithm of the squared standardized residuals for various distributional assumptions on the innovations sequence and alternative values of the GARCH parameters. Given that the $\operatorname{GARCH}(1,1)$ model is versatile enough to produce a range of stochastic processes, with very different moment and memory characteristics, depending on the parameter settings and the distribution of the innovations, we shall be able to examine cases that violate some of De Lima's conditions. Indeed, the $\mathrm{GARCH}(1,1)$ model provides a flexible framework allowing the researcher to control for the amount of temporal dependence, the degree of time-heterogeneity, and the number of unconditional moments that characterize the process by simply changing the values of the model parameters and/or the distributional assumption on the innovations.

Our simulation results suggest that the BDS test performs remarkably well even in cases where De Lima's conditions concerning the memory, moment and time heterogeneity properties of the series of interest fail to hold. This would suggest that some of De Lima's conditions are stricter than necessary. It also intimates that the performance of the BDS test is closely related to the quality of the Quasi Maximum Likelihood (QML) estimator of the conditional variance parameters. In fact, the cases when the distributional divergence of the BDS test from $N(0,1)$ is maximized coincide with those when the bias of the QML estimator is maximized (i.e., when the sample size is less than 250). This association suggests that the most important of De Lima's conditions is the one that requires consistency of the QML estimator. Fortunately for the behaviour of the BDS test, the consistency of QML estimator is preserved even if the GARCH process is neither strictly stationary nor ergodic, having no unconditional moments and being driven by leptokurtic and/or asymmetric innovations (Jensen and Rahbek (2003)).

The paper is structured as follows. Section 2 briefly summarizes the conditions that must be satisfied by the process of interest in order for the test to be nuisance-parameter free. Section 3 outlines the moment, memory and heterogeneity properties of the $\operatorname{GARCH}(1,1)$ model. In addition, it discusses the issue of consistently estimating its parameters, including the case where non-Gaussian innovations drive the GARCH process. Section 4 describes the Monte Carlo setup and reports the main findings. Finally, Section 5 offers a brief summary of the main results and 


\section{The Brock, Dechert and Scheinkman (BDS) test}

We begin by giving a brief description of the BDS test. ${ }^{1}$ Let $\left\{u_{t}\right\}$ be a real-valued scalar stochastic process with the m-history process defined as $u_{t}^{m}=\left(u_{t}, u_{t+1}, \ldots u_{t+m-1}\right)$. The correlation integral at embedding dimension, $\mathrm{m}$, for $\varepsilon>0$, is estimated by the following U-statistic:

$$
C_{m, \varepsilon}=\left(\frac{1}{\left(\begin{array}{c}
\bar{T} \\
2
\end{array}\right)}\right) \sum_{1 \leq s \leq t \leq \bar{T}} \sum I_{\varepsilon}\left(u_{t}^{m}, u_{s}^{m}\right)
$$

where $\bar{T}=T-(M-1), I_{\varepsilon}(.,$.$) is the symmetric indicator kernel with I_{\varepsilon}(z, w)=1$ if $\|z-w\|<\varepsilon$ and 0 otherwise and $\|$. $\|$ is the max-norm. If $\left\{u_{t}\right\}$ is an i.i.d. process with a nondegenerate cumulative distribution $F$, then for fixed $\varepsilon>0$ and $m=1,2, \ldots, C_{m, \varepsilon} \rightarrow C(\varepsilon)^{m}$, as $T \rightarrow \infty$, with probability one, where

$$
C(\varepsilon)=\int[F(z+\varepsilon)-F(z-\varepsilon)] d F(z)
$$

Brock, Dechert and Scheinkman (1987) define the BDS statistic

$$
V_{m, \varepsilon}=\sqrt{T} \frac{C_{m, \varepsilon}-C(\varepsilon)^{m}}{s_{m, \varepsilon}}
$$

where $s_{m, \varepsilon}$ is a consistent estimator of the asymptotic standard deviation, $\sigma_{m, \varepsilon}$, of $\sqrt{T}\left(C_{m, \varepsilon}-C(\varepsilon)^{m}\right)$, and show that under the null hypothesis that $\left\{u_{t}\right\}$ is i.i.d., $V_{m, \varepsilon} \rightarrow N(0,1), \forall \varepsilon>0$ and $m=2,3, \ldots .^{2}$ Initially, the test was designed to be applied to raw series in order to test whether they are i.i.d. It was soon realized that it could also be applied to the estimated residuals from a model to test for omitted dynamics, i.e. to test model adequacy. Brock and Dechert (1988) and De Lima (1996) examined the conditions under which the BDS statistic is nuisance-parameter free, that is its asymptotic distribution does not change when it is applied to the estimated residuals from a model, rather than the raw series itself. The invariance property of the BDS statistic is ensured by a set of sufficient conditions that are more stringent than in the case of smooth U-statistics (the BDS test being a function of those). This is because the indicator kernel, $I_{\varepsilon}(.,$.$) ,$ used in the definition of the correlation integral, is not a differentiable smooth kernel. As a result, special sufficient conditions, ensuring the reversibility between the operations of differentiation and taking the limiting mean, are required in order to guarantee the invariance property of the BDS statistic. Specifically, following Randles (1982), De Lima (1996) derives five sufficient conditions (Assumptions A-D, pp. 240-241, and Assumption E, pp. 245) that ensure the 'nuisance-parameter free' property of the BDS test for linear additive models, such as $y_{t}=G\left(Y_{t-1} ; \theta\right)+e_{t}$, or for models that can be transformed into this format. In this paper, we focus on the effects of the failure of some of these conditions on the invariance property of the BDS test. Assumption A requires $y_{t}$ to be a strong mixing process with summable mixing coefficients, $a(k)$, that is $\sum_{k=1}^{\infty} a(k)^{1 / 2}<\infty$. Assumption B and C impose moment conditions on the difference between the kernel $I_{\varepsilon}$ evaluated at two different points of the residual function. Of these two assumptions, $\mathrm{B}$ is the most important one, and may be verified in a case by case framework by evaluating the supremum of the relevant random variable. Assumption $\mathrm{C}$ is automatically satisfied (by assumption $\mathrm{B}$ ) for bounded

\footnotetext{
${ }^{1} \mathrm{~A}$ detailed description of the BDS test can be found in Brock, Dechert and Scheinkman (1987) or De Lima (1996).

${ }^{2}$ Note that the asymptotic normality of the BDS statistic is established without assuming the existence of any unconditional moments. This feature differentiates the BDS from other nonlinearity tests such as the McLeod-Li test, which requires finite unconditional moments of the fourth order (see McLeod and Li (1983)) or the bispectrum test of Subba Rao and Gabr (1980), which requires finite unconditional moments of the sixth order.
} 
kernels. Assumption D requires the parameters, $\theta$, of the model to be consistently estimated. The consistency of the estimator of $\theta$ is usually based on some memory restrictions on the errors of the model, such as strong mixing with summable mixing coefficients. Of course, if the errors are i.i.d. these restrictions are automatically satisfied. Moreover, for the same reason (consistent estimation of $\theta$ ) moment conditions on the error term (or sometimes on the raw series itself) should be imposed. Therefore, the nuisance-parameter free property of the BDS does require moment restrictions when the BDS test is applied to estimated residuals as opposed to raw data. Finally, Assumption E requires the distribution of the innovations to be absolutely continuous and differentiable, with a bounded density function.

\section{Consistent Estimation and Moment and Memory Properties of the $\operatorname{GARCH}(1,1)$ Process}

We shall investigate the necessity of these conditions (A-E) in the context of a martingaledifference (MD) GARCH(1,1) model. Specifically, we assume that $u_{t}$ is generated as follows:

$$
\begin{gathered}
u_{t}=h_{t} z_{t} \\
z_{t} \sim i . i . d .(0,1)
\end{gathered}
$$

where

$$
h_{t}^{2}=\omega+\mu u_{t-1}^{2}+\delta h_{t-1}^{2}
$$

denotes the variance of $u_{t}$ conditional on the $\sigma$-field, $F_{t-1}$, generated by all information available at time t-1. Note that no specific distributional characteristics of $z_{t}$ have been assumed yet, other than the variance of the unspecified distribution is equal to one. The unit variance of $z_{t}$ is necessary for $h_{t}^{2}$ to be the conditional variance of $u_{t}$. The choice of this particular process for $u_{t}$ was made for two reasons. First, the MD-GARCH $(1,1)$ process is a versatile stochastic process, which, depending on the distribution of the innovations process, $z_{t}$, and the values of the coefficients $\mu$ and $\delta$, is able to reproduce processes that range from $b$-mixing with finite unconditional moments of the fourth order to processes that are neither mixing nor possess any unconditional moments. Note that the error term in (3) is not additive. However, this does not cause any serious difficulties since, by raising both sides of (3) to the square power and taking logs, we can transform (3) into a model that contains an additive error, $\nu_{t}=\ln z_{t}^{2}=\ln u_{t}^{2}-\ln h_{t}^{2}$. The asymptotic distribution of the BDS test applied to the estimated residuals $\widehat{\nu}_{t}$ is the same as that of the test when applied to $\nu_{t}$ provided that $\widehat{\nu}_{t}$ is a consistent estimator of $\nu_{t}$. Many studies, including the original Brock et al. (1991), examine the finite-sample properties of the BDS test by utilizing the non-transformed residuals $\widehat{z}_{t}$ instead of the logged residuals $\widehat{\nu}_{t}$. This in effect means that the model has not been transformed into one containing an additive error term, which has some important implications for the size-properties of the BDS test in finite samples (see next section for a thorough discussion on this point).

As already mentioned, the moment and memory characteristics of $u_{t}$ depend on a) the distribution of the innovations $z_{t}$ and $\mathrm{b}$ ) the values of the parameters $\mu$ and $\delta$. The following subsection summarizes the main theoretical results found in the literature. 


\subsection{Moment and Memory Properties of the GARCH(1,1) Process}

\section{Case I: Fourth-order stationary process.}

He and Terasvirta (1999) provide a necessary and sufficient condition for $E\left(u_{t}^{4}\right)<\infty .{ }^{3}$ This condition requires minimum restrictions on the innovation process $z_{t}$, which is assumed to be a zero mean i.i.d. process with second and fourth unconditional moments denoted by $v_{2}$ and $v_{4}$ respectively. The condition is

$$
\mu^{2} v_{4}+2 \mu \delta v_{2}+\delta^{2}<1
$$

which, under the assumption that $z_{t} \sim N(0,1)$, reduces to Bollerslev's (1986) condition

$$
3 \mu^{2}+2 \mu \delta+\delta^{2}<1
$$

As for the memory properties, Davidson (2002) retains the unit variance assumption $\left(v_{2}=1\right)$ and demonstrates that condition (6) is necessary and nearly sufficient for $u_{t}$ to be near-epoch dependent on $z_{t}$ in $L_{2}$-norm $\left(L_{2}-N E D\right){ }^{4}$ This is sufficient for the series to obey the central limit theorem or the invariance principle.

\section{Case II: Covariance stationary process.}

In this case, condition (6) is violated, but we assume that the unconditional second moment exists, which amounts to

$$
\mu v_{2}+\delta<1
$$

Under the unit variance assumption, the necessary and sufficient condition for covariance stationarity reduces to the well-known condition

$$
\mu+\delta<1
$$

Davidson (2002) proves that, under (9), $u_{t}$ is $L_{1}-\mathrm{NED}$ on $z_{t}$, that is the sufficient condition for the series to obey the law of large numbers, but not the corresponding one for the Central Limit Theorem (CLT). Nevertheless, as shown by De Lima (1996), since covariance stationarity holds, the condition for assumption B is satisfied. Moreover, Carrasco and Chen (2002) prove that (9) is necessary and sufficient for $u_{t}$ to be $b$-mixing. Since $b$-mixing is stronger than $a$-mixing (but weaker than $\varphi$-mixing), we may conclude that condition (9) is sufficient to guarantee the required memory property.

Case III: IGARCH process, with $E\left(u_{t}\right)=0$.

Nelson (1990) proves that a necessary and sufficient condition for $u_{t}$ to be strictly stationary and ergodic is given by

$$
E\left[\ln \left(\delta+\mu z^{2}\right)\right]<0
$$

regardless of the distribution of the i.i.d. innovations $z_{t}$. This condition holds even if

$$
\mu v_{2}+\delta=1
$$

Under (11), $E\left(u_{t}\right)=0$, and $\operatorname{Var}\left(u_{t}\right)=\infty$. In such a case, the $L_{2}-$ NED measure of memory is unavailable, since the unconditional second moment of $u_{t}$ does not exist. Moreover, there is no guarantee that $u_{t}$ is $a$-mixing.

\footnotetext{
${ }^{3}$ See also Ling and McAleer (2002) for the necessary and sufficient condition for the existence of higher order moments of the $\operatorname{GARCH}(\mathrm{r}, \mathrm{s})$ model.

${ }^{4}$ Davidson (2002) shows that the fourth moment condition is necessary and nearly sufficient for the $L_{2}-\mathrm{NED}$ property, regardless of the distribution of the innovations process. The $L_{2}-\mathrm{NED}$ property of a GARCH(1,1) process was first proved by Hansen (1991) under the additional assumption of normality of $z_{t}$.
} 
Case IV: Mildly explosive process, with $E\left(u_{t}\right)=0$.

Nelson (1990) shows that there might be an area in the $(\mu-\delta)$ plane for which $\mu v_{2}+\delta>1$ and condition (10) holds. In such a case, $u_{t}$ is mildly explosive but still strictly stationary and ergodic. If, in addition,

$$
E\left(\delta+\mu z^{2}\right)^{1 / 2}<1
$$

the process $u_{t}$ has an unconditional mean equal to zero.

Case V: Mildly explosive process, with $E\left(u_{t}\right)=\infty$.

This is a case where $\mu$ and $\delta$ are such that (10) is satisfied but (12) fails. By comparing this case with the previous one, one can discern the importance of the finite (zero) mean property of $u_{t}$ for the properties of the BDS statistic.

Let us now turn to the problem of estimating the conditional variance parameters, $\theta=(\omega, \mu, \delta)$.

\subsection{Consistent Estimation of the $\operatorname{GARCH}(1,1)$ parameters.}

When $z_{t} \sim N(0,1)$, the employment of the Gaussian likelihood function results in Maximum Likelihood (ML) estimates, $\widehat{\theta}_{M L}$, of $\theta=(\omega, \mu, \delta)$. If the distribution of $z_{t}$ is not Normal, then the Gaussian likelihood function produces the so-called Quasi Maximum Likelihood (QML) estimates of $\theta$. The asymptotic properties of the QML estimates of $\theta$ have been studied in Bollerslev and Wooldridge (1992), Lumsdaine (1996) and Lee and Hansen (1994). All these studies require the validity of (10), that is they assume that $u_{t}$ is strictly stationary and ergodic so that the relevant laws of large numbers apply. They differ in the moment restrictions that they impose either on $u_{t}$ or on $z_{t}$. More recently, Jensen and Rahbek (2003) relax the stationarity condition (10) and prove the $\sqrt{T-}$ consistency (and asymptotic normality) of the QML estimates in cases where $u_{t}$ is a nonstationary process with unbounded low order moments. The only condition they impose is the existence of the fourth moment of $z_{t}$ (not $u_{t}$ ). Interestingly, they claim that unreported simulation evidence suggests that the rate of convergence is faster in the non-stationary than in the stationary

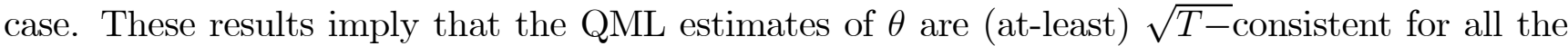
five cases defined above provided that $\nu_{4}<\infty$. However, as will be shown below, $\sqrt{T-}$ consistency of the QML estimates of $\theta$ seems to be achieved even in cases where $\nu_{4}$ is infinite, as for example when $z_{t}$ follows a $t(4)-$ or $t(3)$-distribution. Therefore, as a by-product of this study, we provide evidence that the existing conditions for $\sqrt{T-}$ consistency of the QML estimates of $\theta$ may be relaxed.

\section{Monte Carlo Simulations}

In this section we carry out Monte Carlo simulations that aim at examining the distribution of the BDS statistic and the resulting performance of the BDS test in cases where some of the sufficient conditions for the invariance property of the BDS test fail. Since the performance of the BDS test statistic is closely linked to the quality of the estimation of the GARCH parameters, we examine both issues jointly. The simulations are designed as follows. First, we choose the distribution of the innovation $z_{t}$, and generate i.i.d. series with mean zero and variance one. Second, we select the GARCH parameters, $\delta$ and $\mu$, in such a way as to generate a series $u_{t}$ with specific moment and memory properties as outlined in cases I to V. Third, we estimate a GARCH $(1,1)$ model, take the logarithm of the squared standardized residuals and compute the BDS statistic for various values of $m$ and $\varepsilon / \sigma$ ( $\sigma$ being the standard deviation of the sample series) and for alternative sample sizes, $T$. In particular, the values of $\varepsilon / \sigma$ considered are $0.5,1,1.5$ and 2 , those of $m$ are $2,3, \ldots 10$, and the sample sizes are $100,250,500,1000$ and $2000 . .^{5}$ The values of these parameters

\footnotetext{
${ }^{5}$ Initially, we generate a sample of $T+250$ observations and discard the first 250 to account for the effect of the initial conditions.
} 
were selected so as to make our results comparable to those of the existing literature, in particular those in Brock et al. (1991), Brooks and Heravi (1999) and Brooks and Henry (2000). We repeat this procedure 2000 times and calculate the mean, variance, skewness and kurtosis coefficients of the BDS statistic. We also compute the $5 \%$ empirical size. In order to be able to compare our results with those from other studies, which have applied the BDS test on the non-logged residuals, we repeat the above described procedure for the case where the statistic, denoted as $B D S^{*}$, is calculated on the basis of the non-logged standardized residuals. Further, for each parameter configuration and for each sample size, we compute the average absolute bias of the QML estimates of $\omega, \mu$ and $\delta$. This has two aims: First, to examine the behaviour of the QML estimator of the GARCH parameters in small samples when the requirement $\nu_{4}<\infty$ is satisfied. Although consistency holds in this case (i.e. when $\nu_{4}<\infty$ ), the bias of the QML estimator, in small samples, may affect the behaviour of the BDS test. Second, and more importantly, to examine whether consistency is threatened in cases that fall outside the spectrum covered by the analytic results of Jensen and Rahbek (2003), that is, for example, when $z_{t}$ follows a $t(4)-$ or $t(3)$-distribution. Although we cannot reliably establish consistency results (or identify rates of convergence) by means of simulation techniques, the simulation results can nevertheless give an indication of whether problems might arise when the fourth moment of $z_{t}$ does not exist.

\subsection{The case of Gaussian innovations}

First, we assume that $z_{t}$ is an i.i.d. Gaussian process. Figure 1 shows the regions in the $(\mu-\delta)$ plane that correspond to the five cases defined above, when $z_{t} \sim N(0,1)$. Regions 1 and 2 correspond to cases I and II respectively; the boundary between regions 2 and 4 to case III; and finally regions 4 and 5 to cases IV and V respectively. We selected values of $(\mu, \delta)$ ranging from the upper left area of each region to the bottom right one, in order to achieve full coverage of each region. ${ }^{6}$ However, we report in the Tables only the results for one pair of values of $(\mu, \delta)$, which is representative of those typical of the relevant literature, i.e. a very small $\mu$ and a much larger $\delta$. We mention in the text any cases where other points from the same region produce different results. The reported results are those for the following pairs of values for $(\mu, \delta)$ corresponding to the five regions mentioned above:

Model I: $(\mu, \delta)=(0.1,0.85)$

Model II: $(\mu, \delta)=(0.1,0.895)$

Model III: $(\mu, \delta)=(0.1,0.9)$

Model IV: $(\mu, \delta)=(0.1,0.904)$

Model $\mathrm{V}:(\mu, \delta)=(0.1,0.907)$

Before presenting the simulation evidence on the performance of the BDS test statistic, let us first discuss the ML estimates of the GARCH parameters for each of the five models defined above. Note that, since $z_{t}$ is Gaussian, in view of the analytical results of Jensen and Rahbek (2003), there is no reason to believe that consistency is threatened in any of these cases. Panel A of Table 1 reports the mean absolute bias of the ML estimates of $\mu$ and $\delta$ for Models I to V. ${ }^{7}$ This evidence confirms the theoretical results, suggesting that the ML estimates of $\mu$ and $\delta$ are consistent, regardless of whether the process $u_{t}$ is stationary or possesses any moments. More interestingly, the bias of both $\mu$ and $\delta$ is decreasing as we move from region 1 to region 5 for almost all the sample sizes considered, especially so for $T=100 .^{8}$ This seems to support the Jensen and

\footnotetext{
${ }^{6}$ Throughout the simulations we set the constant term $\omega$ such that the unconditional variances (where they are defined) are the same across different simulations.

${ }^{7}$ We also report the mean absolute bias of the QML estimates as a percentage of the true value of the parameters (Panel B of Table 1) in order to facilitate the comparison between the results of the alternative distributions considered in the simulation.

${ }^{8}$ The standard deviation of the ML estimates of the GARCH parameters decreases with the persistence of the process. The results are not reported to save space.
} 
Rahbek (2003) claim that the rate of convergence of the ML estimates seems to increase as we move towards the nonstationary region. However, the finite sample bias of the ML estimates (especially for $T=100$ ) may influence the distribution and the size properties of BDS.

We now turn to the main focus of our analysis, that is the behaviour of the BDS test, applied to the logged squared standardized residuals. As mentioned earlier, we also examine the behaviour of the $B D S^{*}$ test applied to the untransformed squared residuals for comparison with the existing results from the literature, and in particular with those in Brock et al. (1991). For brevity we report in Table 2 only the results for $m=2,5$ and 8 (the full set of results is available upon request). Those for the $B D S^{*}$ test are in the parentheses under the corresponding ones for the $B D S$ test. In summary, we find the following:

1. When the non-logged standardized residuals are used, the distribution of the $B D S^{*}$ is substantially different from the $N(0,1)$ distribution. More specifically, it always has a negative mean, while the standard deviation is less than unity in most cases. In addition, it is positively skewned and leptokurtic, especially for large values of $m$. Moreover, it moves away from the $N(0,1)$ distribution as the sample size increases - this can be clearly seen in Figure 2, which shows the distribution of the $B D S^{*}$ test for different sample sizes. As a result, the performance of the $B D S^{*}$ test is very poor. In general, it is under-sized and seems to become more conservative as the sample size increases. This is the case for all the values of $m$ considered and for all the values of $\varepsilon / \sigma$ except for $\varepsilon / \sigma=0.5$, when the $B D S^{*}$ test appears to be heavily over-sized for large $m$, even in large samples. This picture is qualitative similar to the one emerging from previous studies (Brock et al. (1991) and Brooks and Henry (2000)). In addition, we observe that the size-distortions remain the same throughout the $(\mu, \delta)$ parameter space, ranging from region 1 to region 5 (previous studies examined only the case where fourth order stationarity holds).

2.When the log-transformation is implemented, the picture changes dramatically. As far as the distribution of the BDS test is concerned, it transpires from Table 3 that it tends to the standard normal distribution as the sample size increases. ${ }^{9}$ This is more evident in Figure 3, which shows the distribution of the $B D S$ test for different sample sizes (region 1): it is clear that it approaches the $N(0,1)$ distribution as $T$ increases (for $T=1000$ the two distributions are almost identical). However, for small sample sizes we observe a negative mean and a standard deviation which is larger than unity. The distribution is also positively skewed and leptokurtic for large values of $m$. This divergence between the distribution of the BDS test and the $N(0,1)$ seems to be caused by the relatively substantial bias of the ML estimates for $T=100$. Moreover, the moment properties of the process do not seem to affect the the distribution of the $B D S$ test. Figure 4 shows it for the first and fifth region $(T=1000)$. In both cases it is very close to $N(0,1)$. As a result of these distributional characteristics, the $B D S$ test does not suffer from any serious size distortions for sample sizes $T \geq 250$ and for any values of $m$ and $\varepsilon / \sigma$ (except for the small value $\varepsilon / \sigma=0.5$ ).

In brief, the simulation results suggest that some of the assumptions used by De Lima to derive the nuisance-parameter free property of the $B D S$ test may not be necessary. More specifically, the assumption that $z_{t} \sim N(0,1)$ ensures that, for all the five regions considered, the consistency of the ML estimates of the GARCH parameters is preserved, that is Assumption D is satisfied throughout the simulations, and so is Assumption E. Similarly, Assumption C is satisfied in all cases. However, De Lima assumes second order stationarity (i.e. $\mu+\delta<1$ ) to ensure that Assumption B holds. In the case of covariance stationarity, so does Assumption A. If however, $\mu+\delta \geq 1$, then these assumptions (A and B) might not be valid, in fact Assumption $\mathrm{A}$ is violated, since the process is not $b$ - mixing. Our simulation results, however, suggest that the validity of these assumptions is not necessary for the BDS test to be nuisance-parameter free. In fact, its

\footnotetext{
${ }^{9}$ For brevity, we report only the results for $\varepsilon / \sigma=1$ and 1.5. All the other results are available on request.
} 
distribution is close to $N(0,1)$ even in cases that belong to region 5 , that is for processes that have no unconditional moments and for which the memory condition is far from being satisfied. As we can see in Table 3 and Figure 4, in the case of a mildly explosive process with infinite mean (i.e. region 5) the distribution of the $B D S$ test is similar to that of a process with finite fourth moments (i.e. region 1), and the test does not suffer from any size distortions. On the other hand, any distributional divergence from $N(0,1)$ is associated with a relatively substantial bias of the ML estimator, which in turn implies that the good quality of these estimates is very important for the behaviour of the BDS test.

So far, the assumption of Gaussian innovations ensures the finiteness of the fourth moment of $z_{t}$, which is a sufficient condition for the consistency of the QML estimator of the GARCH parameters (Jensen and Rahbek (2003)). In the following subsection, we relax the assumption of a finite fourth moment of the innovation term by allowing $z_{t}$ to follow a Student's t-distribution with 3 and 4 degrees of freedom. In this way, we test the necessity of this assumption to achieve consistency of the QML estimator. If this is lost, Assumption D of De Lima (1996) is violated and the nuissance-parameter free property of BDS may not hold.

\subsection{Symmetric, heavy-tailed distributions: The case of t-innovations}

In this set of experiments, we assume that the innovations $z_{t}$ follow a standardized t-distribution with $\kappa$ degrees of freedom, that is $z_{t}=\left(\xi_{t} / \sqrt{k / k-2}\right)$, where $\xi_{t}$ is an i.i.d. $t(k)$ variate. The new distributional assumption implies that some of the aforementioned cases (I) to (V) are no longer defined. Indeed, the number of valid cases, under t-innovations, depends on the degreesof-freedom parameter, $k$. In particular, for $k=5$, all the cases (I)-(V) exist. For $k=4$ and $k=3$, fourth moments of the innovations do not exist, which in turn implies that case I is not defined. As their finiteness was the condition imposed by Jensen and Rahbek (2003) to prove the $\sqrt{T-}$ consistency of the QML estimates, it is clearly of interest to examine the properties of such estimates and of the BDS statistic under these circumstances. Figure 5 defines the corresponding cases 2 to 5 for $k=4$.

We first investigate the consistency property of the QML estimates of $\theta$ when $z_{t}$ follows a standardized $t(3)$ - or $t(4)$-distribution. In general, the results are similar for the two distributions; therefore, we only report the results for the latter. We consider values of $(\mu, \delta)$ corresponding to case II to V, and, once again, we report the results for only one pair of values for each region. More specifically, the models under investigation are the following:

Model VI: $(\mu, \delta)=(0.1,0.8)$

Model VII: $(\mu, \delta)=(0.1,0.9)$

Model IIX: $(\mu, \delta)=(0.1,0.91)$

Model IX: $(\mu, \delta)=(0.1,0.915)$

The mean absolute bias of the GARCH parameters for these four models is given in Table 1 (fifth and sixth column). It clearly decreases with the sample size. Therefore, consistency seems to hold even though the innovations do not possess a finite fourth moment. In other words, the simulations provide evidence that the existing conditions for $\sqrt{T-}$ consistency of the QML estimates of $\theta$ may be relaxed. ${ }^{10}$ Similarly to the Gaussian case, the bias of both $\mu$ and $\delta$ decreases as we move from region 2 to region 5 for almost all the sample sizes considered. Once again, convergence seems to be faster for the mildly explosive processes (that is for regions 4 and 5). Finally, the bias and the standard deviation of the estimates are higher in the case of the $t(3)$ - distribution compared to the one of the $t(4)$-distribution.

\footnotetext{
${ }^{10}$ It is important to note that Jensen and Rahbek (2003) assume a finite fourth moment of the innovation $z_{t}$ to establish both consistency and normality of the QML estimator of the GARCH parameters. Our results indicate that this assumption may be unnecessarily strict to achieve consistency. Lee and Hansen (1994, Theorem 2, pp. 37) prove the consistency of the QML estimator by assuming finiteness of the second moment of $z_{t}$. However, their proof is based on the additional assumption that $\mu+\delta \lesseqgtr 1$.
} 
We now examine the size properties of the BDS statistic. The results for both $B D S$ and $B D S^{*}$ are presented in Table 4, and, on the whole, are similar to those for the Gaussian case. That is, the $B D S^{*}$ test is in general undersized and it becomes more conservative as the sample size increases. Once again, for $\varepsilon / \sigma=0.5$ and for large $m$, it is heavily oversized. On the other hand, the implementation of the log-transformation restores the good size properties of the test, especially for $T \geq 500$ and $\varepsilon / \sigma=1$ and $1.5 .^{11}$ The size behaviour of the $B D S$ (and $B D S^{*}$ ) test reflects the distributional features of these statistics. Figure 6 presents its distribution for $T=100$ and 500. It is clear that this gets closer to $N(0,1)$ as the sample size increases. However, it is now often more leptokurtic than in the case of Gaussian innovations. Furthermore, for large values of $m$, it is positively skewned. For example, for the configuration $(\varepsilon / \sigma, m, T)=(1,8,100)$ and for the IGARCH process, its skewness and kurtosis when $z_{t} \sim t(4)$ is 1.17 and 6.26 respectively, compared to 0.91 and 4.71 in the Gaussian case. These minor distortions in the distribution of the test are again associated with the relatively higher biases observed in the QML estimates of the GARCH parameters. Finally, the behaviour of the test is invariant to the moment and memory characteristics of the process.

In summary, in the four models considered in this second set of simulations, the validity of De Lima's conditions is guaranteed only for model VI. More specifically, in this case $\mu+\delta<1$, suggesting that Assumptions A and B hold. Moreover, the QML estimator is consistent (see Theorem 2, pp. 37, in Lee and Hansen(1994)). Finally, Assumptions C and E are also valid. On the other hand, the other three models considered violate Assumption A and Assumption B is not guaranteed. Furthermore, the consistency of the QML estimates (Assumption D) is not certain, since $\mu+\delta \geq 1$ and the innovations do not possess finite fourth moments. Therefore, the nuisance-parameter free property of the BDS test is threatened for models VII, IIX and IX. However, the simulation results indicate that it approximates well the $N(0,1)$ distribution for $T \geq 500$, suggesting that De Lima's conditions may be unnecessarily strict. Similarly, it appears that the existing conditions for the consistency of the QML estimates may be relaxed. It is important to note that although consistency of the QML estimator seems to holds, the small sample $(T \leq 250)$ bias of the estimates is now higher compared to the Gaussian case, resulting in greater small size distortions of the BDS test.

\subsection{Asymmetric Distributions: The case of $\chi^{2}$ innovations}

So far, we have considered innovations that follow a symmetric distribution. We now analyze the behaviour of the BDS statistic when the innovations $z_{t}$ have an asymmetric distribution. Asymptotic theory suggests that this does not affect the consistency of the QML estimator. However, it might affect the finite sample properties of the estimates. To shed some light on this issue, we carry out some additional simulations in which the innovations $z_{t}$ follow a standardized $\chi^{2}$-distribution, that is $z_{t}=\frac{\xi_{t}-1}{\sqrt{2}}$, where $\xi_{t}$ is an i.i.d. $\chi^{2}(1)$ variate. Under this assumption, all five cases (I to V) are meaningful. Figure 7 shows the corresponding regions in the $(\mu, \delta)$ plane.

We consider the following models:

Model X: $(\mu, \delta)=(0.1,0.8)$

Model XI: $(\mu, \delta)=(0.1,0.85)$

Model XII: $(\mu, \delta)=(0.1,0.9)$

Model XIIV: $(\mu, \delta)=(0.1,0.91)$

Model XIV: $(\mu, \delta)=(0.1,0.92)$

Table 1 (columns 7 and 8) contains the mean absolute bias of the estimates of $\mu$ and $\delta$ for these models. As before, the mean absolute bias of the QML estimators decreases with the sample size and with the persistence of the process. It is much higher compared to the Gaussian case for all the

\footnotetext{
${ }^{11}$ In the case of the $t(3)$-distribution, the sizes of the $B D S$ test are about 1 to 2 percentage points higher than those of the $t(4)$-distribution.
} 
sample sizes considered. However, the rate of convergence in the case of the $\chi^{2}$-distribution looks faster than that in the other distributions considered. This is evident in Figure 8, which presents the mean absolute bias of the QML estimator of $\mu$ for different sample sizes and distributions. For small samples, the bias in the case of the $\chi^{2}$-distribution is higher than that in the case of the $t(3)$ - or $t(4)$-distribution. However, as the sample size increases, it becomes similar or even lower than in the case of the $t(3)$ - or $t(4)$-distribution, due to the faster rate of convergence. It seems that the asymmetric distribution of $z_{t}$ has a negative effect on the accuracy of the estimates of the GARCH parameters in small samples. Nevertheless, the QML estimator remains consistent.

The results for the size properties of the $B D S$ and $B D S^{*}$ tests are presented in Table 5 . Although the bias of the QML estimators is larger in the case of the $\chi^{2}$ compared to that of the Normal distribution (especially in small samples), the size of the $B D S$ test is similar in the two cases. However, its good size properties are deceptive. A thorough investigation of its distribution reveals this differs substantially from the standard normal for $T \leq 250$. More specifically, it has a positive mean and is also positively skewed and leptokurtic. As a result, the majority of the rejections of the null hypothesis are right-sided percentage rejections. For example, for the configuration $(\varepsilon / \sigma, m, T)=(1,5,100)$ and for the IGARCH process, the BDS test produces a value greater than 1.96 at a frequency of 6.45 percent. On the other hand, the left-sided percentage rejections are only 1.50. Once again, we find evidence of a close association between the bias of the QML estimates and the behaviour of the BDS test. Nevertheless, the distribution of the statistic approaches $N(0,1)$ as the sample size increases (given that $\varepsilon / \sigma \geq 1$ ).

\section{Conclusions}

In this study we carry out Monte Carlo simulations to examine the behaviour of the widely used Brock, Dechert and Scheinkman (BDS) test when applied to either the standardized residuals or the logs of the standardized residuals of an estimated $\operatorname{GARCH}(1,1)$ model as a test for the adequacy of this specification. More in detail, we consider a variety of distributions for the innovations (implying different moment and memory characteristics of the error term) to examine the "necessity" of De Lima's (1996) conditions for the invariance property of the BDS test. Furthermore, we investigate the finite sample performance of the BDS test. As a by-product, we examine the related issues of consistency and small sample bias of the QML estimators of the GARCH parameters.

The results indicate that the BDS test is generally well-behaved when applied to the logarithm of the standardized residuals of a $\operatorname{GARCH}(1,1)$ model. Its distribution approximates the standard normal distribution for $T \geq 1000$ and we do not observe any size distortions for large samples. Moreover, the behaviour of the BDS test does not seem to be affected by the moment properties of the series under scrutiny. Its distribution tends to $N(0,1)$ even for mildly explosive processes that violate some of De Lima's (1996) conditions for the nuissance-parameter free property of the BDS test. Thus, our results suggest that De Lima's (1996) assumptions on mixing and covariance stationarity to prove the nuissance-parameter free property of the BDS test may be unnecessarily strict.

As far as the small sample behaviour of BDS is concerned, the simulations reveal that this is closely related to the finite sample performance of the QML estimator of the GARCH parameters. More specifically, the small sample bias of the QML estimator (especially for $T \leq 250$ ) results in (small) size distortions of the BDS test. These are due to the skewed and leptokurtic distribution that the BDS test has in such small samples. The small sample bias of the GARCH estimates is greater when the innovation term of the process under examination follows a leptokurtic or an asymmetric distribution. In such cases, the distribution of BDS needs larger sample sizes to approximate well the $N(0,1)$ distribution. Finally, the consistency of the QML estimator of the GARCH parameters seems to hold in all the cases considered, even when the Jensen's and 
Rahbek's (2003) condition that the innovation term should possess a finite fourth moment is violated. Therefore, we provide evidence that the existing conditions for $\sqrt{T-}$ consistency of the QML estimates of the GARCH parameters can be relaxed.

\section{References}

[1]Bollerslev, T., 1986. Generalized Autoregressive Conditional Heteroskedasticity. Journal of Econometrics 31, 307-327.

[2]Bollerslev, T. and J.M. Wooldridge, 1992. Quasi-Maximum Likelihood Estimation and Inference in Dynamic Models with Time Varying Covariances. Econometric Reviews 11, 143-172.

[3]Brock, W.A., W.D. Dechert and J. Scheinkman, 1987. A Test for Independence Based on the Correlation Dimension. Department of Economics, University of Wisconsin, University of Houston and University of Chicago (Revised Version, 1991: Brock, W.A., W.D. Dechert, J. Scheinkman and B. LeBaron).

[4]Brock, W.A., W.D. Dechert, 1988. A General Class of Specification Tests: The Scalar Case. Proceedings of the Business and Economic Statistics of the American Statistical Association, 70-79.

[5]Brock, W.A., D.A. Hsieh and B. LeBaron, 1991. Nonlinear Dynamics, Chaos, and Instability: Statistical Theory and Economic Evidence. MIT Press, Cambridge, MA.

[6]Brooks, C. and O.T. Henry, 2000. Can Portmanteau Tests Serve as General Mis-Specification Tests? Evidence from Symmetric and Asymmetric GARCH Models. Economics Letters 67, 245-251.

[7]Brooks, C. and S.M. Heravi, 1999. The Effect of (Mis-Specified) GARCH Filters on the Finite Sample Distribution of the BDS Test. Computational Economics 13, 147-162.

[8]Carrasco, M. and X. Chen, 2002. Mixing and Moment Properties of Various GARCH and Stochastic Volatility Models. Econometric Theory 18, 17-39.

[9]Davidson J., 2002. Establishing Conditions for the Functional Central Limit Theorem in Nonlinear and Semiparametric Time Series Processes. Journal of Econometrics 106, 243-269.

[10]De Lima, P.J.F, 1996. Nuisance Parameter Free Properties of Correlation Integral Based Statistics. Econometric Reviews 15, 237-259.

[11]Engle, R.F., 1982. Autoregressive Conditional Heteroskedasticity with Estimates of the Variance of the United Kingdom Inflation. Econometrica 50, 987-1007.

[12]Hansen, B.E., 1991. Strong Laws for Dependent Heterogeneous Processes. Econometric Theory 7, 213-221.

[13]He, C. and T. Terasvirta, 1999. Fourth moment structure of the GARCH(p,q) process. Econometric Theory 15, 824-846.

[14]Jensen, S.T. and A. Rahbek, 2003. Asymptotic Normality of the QMLE Estimator of ARCH in the Nonstationary case. Econometrica forthcoming.

[15]Lee, S.W and B.E. Hansen, 1994. Asymptotic Theory for the GARCH(1,1) Quasi-maximum Likelihood Estimator. Econometric Theory 10, 29-52. 
[16]Ling, S. and M. McAleer, 2002. Necessary And Sufficient Moment Conditions For The $\operatorname{Garch}(\mathrm{R}, \mathrm{S})$ and Asymmetric Power Garch(R,S) Models. Econometric Theory 18, 3, 722 729.

[17]Lumsdaine, R., 1996. Consistency and Asymptotic Normality of the Quasi-maximum Likelihood Estimator in IGARCH(1,1) and Covariance Stationary GARCH(1,1) Models. Econometrica $64,575-596$.

[18]McLeod, A.I. and W.K. Li, 1983. Diagnostic Checking ARMA Time Series Models Using Squared-Residual Autocorrelations. Journal of Time Sweries Analysis 4, 269-273.

[19] Nelson, D.B, 1990. Stationarity and Persistence in the GARCH(1,1) model. Econometric Theory $6,318-334$.

[20]Randles, R.H., 1982. On the Asymptotic Normality of Statistics with Estimated Parameters. Annals of Statistics 10, 462-474.

[21]Subba Rao, T. and M. Gabr, 1980. A Test for Linearity of Stationary Tiem Series. Journal of Time Series Analysis 1, 145-158. 
Table 1: A: Mean Absolute Bias (MAB) of the QML Estimates

$\mathrm{B}$ : MAB of the QML Estimates (as a percentage of the true parameter value)

\begin{tabular}{|c|c|c|c|c|c|c|c|}
\hline A & & \multicolumn{2}{|c|}{$\mathrm{N}(0,1)$} & \multicolumn{2}{|c|}{$t(4)$} & \multicolumn{2}{|c|}{$x^{2}$} \\
\hline Case & $\begin{array}{c}\text { Sample } \\
\text { Size }\end{array}$ & $\boldsymbol{\mu}$ & $\delta$ & $\mu$ & $\delta$ & $\boldsymbol{\mu}$ & $\delta$ \\
\hline I & $\begin{array}{c}100 \\
250 \\
500 \\
1000 \\
2000 \\
\end{array}$ & $\begin{array}{l}0.072 \\
0.044 \\
0.032 \\
0.022 \\
0.016\end{array}$ & $\begin{array}{l}0.213 \\
0.139 \\
0.101 \\
0.079 \\
0.060\end{array}$ & $\begin{array}{l}-- \\
--- \\
--- \\
--- \\
--- \\
\end{array}$ & $\begin{array}{l}--- \\
--- \\
--- \\
--- \\
--- \\
\end{array}$ & $\begin{array}{l}0.191 \\
0.112 \\
0.075 \\
0.053 \\
0.034\end{array}$ & $\begin{array}{l}0.217 \\
0.168 \\
0.138 \\
0.107 \\
0.068\end{array}$ \\
\hline II & $\begin{array}{c}100 \\
250 \\
500 \\
1000 \\
2000 \\
\end{array}$ & $\begin{array}{l}0.061 \\
0.034 \\
0.022 \\
0.016 \\
0.011\end{array}$ & $\begin{array}{l}0.177 \\
0.060 \\
0.027 \\
0.017 \\
0.011\end{array}$ & $\begin{array}{l}0.135 \\
0.088 \\
0.061 \\
0.048 \\
0.032\end{array}$ & $\begin{array}{l}0.209 \\
0.156 \\
0.121 \\
0.095 \\
0.062 \\
\end{array}$ & $\begin{array}{l}0.189 \\
0.100 \\
0.071 \\
0.047 \\
0.031\end{array}$ & $\begin{array}{l}0.231 \\
0.152 \\
0.107 \\
0.070 \\
0.043\end{array}$ \\
\hline III & $\begin{array}{c}100 \\
250 \\
500 \\
1000 \\
2000 \\
\end{array}$ & $\begin{array}{l}0.060 \\
0.033 \\
0.023 \\
0.014 \\
0.010 \\
\end{array}$ & $\begin{array}{l}0.146 \\
0.053 \\
0.026 \\
0.014 \\
0.010 \\
\end{array}$ & $\begin{array}{l}0.102 \\
0.058 \\
0.041 \\
0.030 \\
0.023\end{array}$ & $\begin{array}{l}0.200 \\
0.082 \\
0.046 \\
0.027 \\
0.019 \\
\end{array}$ & $\begin{array}{l}0.165 \\
0.099 \\
0.058 \\
0.038 \\
0.024\end{array}$ & $\begin{array}{l}0.233 \\
0.118 \\
0.063 \\
0.036 \\
0.021\end{array}$ \\
\hline IV & $\begin{array}{c}100 \\
250 \\
500 \\
1000 \\
2000\end{array}$ & $\begin{array}{l}0.056 \\
0.032 \\
0.022 \\
0.014 \\
0.010 \\
\end{array}$ & $\begin{array}{l}0.135 \\
0.045 \\
0.024 \\
0.014 \\
0.009 \\
\end{array}$ & $\begin{array}{l}0.103 \\
0.055 \\
0.041 \\
0.029 \\
0.020\end{array}$ & $\begin{array}{l}0.193 \\
0.070 \\
0.040 \\
0.024 \\
0.016 \\
\end{array}$ & $\begin{array}{l}0.178 \\
0.099 \\
0.059 \\
0.035 \\
0.022 \\
\end{array}$ & $\begin{array}{l}0.241 \\
0.115 \\
0.057 \\
0.029 \\
0.017 \\
\end{array}$ \\
\hline V & $\begin{array}{c}100 \\
250 \\
500 \\
1000 \\
2000 \\
\end{array}$ & $\begin{array}{l}0.055 \\
0.032 \\
0.022 \\
0.015 \\
0.010\end{array}$ & $\begin{array}{l}0.122 \\
0.041 \\
0.022 \\
0.013 \\
0.009\end{array}$ & $\begin{array}{l}0.100 \\
0.052 \\
0.036 \\
0.027 \\
0.021\end{array}$ & $\begin{array}{l}0.181 \\
0.064 \\
0.034 \\
0.021 \\
0.015\end{array}$ & $\begin{array}{l}0.165 \\
0.091 \\
0.057 \\
0.031 \\
0.020\end{array}$ & $\begin{array}{l}0.234 \\
0.093 \\
0.045 \\
0.022 \\
0.014\end{array}$ \\
\hline B & & \multicolumn{2}{|c|}{$\mathrm{N}(0,1)$} & \multicolumn{2}{|c|}{$t(4)$} & \multicolumn{2}{|c|}{$x^{2}$} \\
\hline Case & $\begin{array}{c}\text { Sample } \\
\text { Size }\end{array}$ & $\mu$ & $\delta$ & $\mu$ & $\delta$ & $\mu$ & $\delta$ \\
\hline I & $\begin{array}{c}100 \\
250 \\
500 \\
1000 \\
2000 \\
\end{array}$ & $\begin{array}{l}72,233 \\
44,468 \\
31,947 \\
22,205 \\
16,124 \\
\end{array}$ & $\begin{array}{c}25,092 \\
16,303 \\
11,878 \\
9,238 \\
7,114 \\
\end{array}$ & $\begin{array}{l}--- \\
--- \\
--- \\
--- \\
--\end{array}$ & $\begin{array}{l}--- \\
--- \\
--- \\
--- \\
---\end{array}$ & $\begin{array}{c}191,246 \\
112,135 \\
74,546 \\
52,619 \\
34,483 \\
\end{array}$ & $\begin{array}{c}27,184 \\
21,017 \\
17,227 \\
13,337 \\
8,451 \\
\end{array}$ \\
\hline II & $\begin{array}{c}100 \\
250 \\
500 \\
1000 \\
2000 \\
\end{array}$ & $\begin{array}{l}60,787 \\
33,964 \\
22,499 \\
15,602 \\
10,959 \\
\end{array}$ & $\begin{array}{c}19,726 \\
6,695 \\
3,042 \\
1,868 \\
1,253 \\
\end{array}$ & $\begin{array}{c}135,110 \\
88,127 \\
60,795 \\
47,784 \\
32,383 \\
\end{array}$ & $\begin{array}{c}26,129 \\
19,528 \\
15,161 \\
11,841 \\
7,725 \\
\end{array}$ & $\begin{array}{c}189,386 \\
100,156 \\
70,663 \\
46,531 \\
31,193 \\
\end{array}$ & $\begin{array}{c}27,208 \\
17,830 \\
12,614 \\
8,187 \\
5,091 \\
\end{array}$ \\
\hline III & $\begin{array}{c}100 \\
250 \\
500 \\
1000 \\
2000 \\
\end{array}$ & $\begin{array}{l}59,962 \\
32,729 \\
22,843 \\
14,114 \\
10,360 \\
\end{array}$ & $\begin{array}{c}16,178 \\
5,837 \\
2,930 \\
1,596 \\
1,107 \\
\end{array}$ & $\begin{array}{c}101,889 \\
58,302 \\
41,470 \\
29,863 \\
23,040 \\
\end{array}$ & $\begin{array}{c}22,215 \\
9,080 \\
5,083 \\
3,015 \\
2,161 \\
\end{array}$ & $\begin{array}{c}165,375 \\
98,690 \\
58,008 \\
38,219 \\
24,055 \\
\end{array}$ & $\begin{array}{c}25,877 \\
13,128 \\
7,035 \\
4,046 \\
2,369 \\
\end{array}$ \\
\hline IV & $\begin{array}{c}100 \\
250 \\
500 \\
1000 \\
2000 \\
\end{array}$ & $\begin{array}{l}56,240 \\
32,435 \\
22,258 \\
14,408 \\
10,113 \\
\end{array}$ & $\begin{array}{c}14,957 \\
5,022 \\
2,609 \\
1,535 \\
1,010 \\
\end{array}$ & $\begin{array}{c}102,562 \\
55,372 \\
41,243 \\
29,206 \\
19,822 \\
\end{array}$ & $\begin{array}{c}21,166 \\
7,654 \\
4,424 \\
2,614 \\
1,708 \\
\end{array}$ & $\begin{array}{c}178,027 \\
98,699 \\
58,879 \\
34,949 \\
22,317 \\
\end{array}$ & $\begin{array}{c}26,492 \\
12,617 \\
6,288 \\
3,166 \\
1,894 \\
\end{array}$ \\
\hline V & $\begin{array}{c}100 \\
250 \\
500 \\
1000 \\
2000 \\
\end{array}$ & $\begin{array}{l}54,582 \\
31,738 \\
22,297 \\
14,559 \\
10,002 \\
\end{array}$ & $\begin{array}{c}13,446 \\
4,547 \\
2,457 \\
1,464 \\
0,986 \\
\end{array}$ & $\begin{array}{l}99,782 \\
52,100 \\
35,714 \\
27,417 \\
20,908 \\
\end{array}$ & $\begin{array}{c}19,796 \\
6,962 \\
3,705 \\
2,289 \\
1,656 \\
\end{array}$ & $\begin{array}{c}164,614 \\
91,151 \\
56,802 \\
31,217 \\
20,246 \\
\end{array}$ & $\begin{array}{c}25,406 \\
10,103 \\
4,879 \\
2,433 \\
1,478 \\
\end{array}$ \\
\hline
\end{tabular}


Table 2: Size of BDS (and BDS* in parentheses) for the $\mathrm{N}(0,1)$ Distribution

\begin{tabular}{|c|c|c|c|c|c|c|c|c|c|c|c|c|c|}
\hline \multirow[b]{2}{*}{ Case } & & \multicolumn{3}{|c|}{$\varepsilon / \sigma=0.5$} & \multicolumn{3}{|c|}{$\varepsilon / \sigma=1$} & \multicolumn{3}{|c|}{$\varepsilon / \sigma=1.5$} & \multicolumn{3}{|c|}{$\varepsilon / \sigma=2$} \\
\hline & $\mathbf{T}$ & 2 & 5 & 8 & 2 & 5 & 8 & 2 & 5 & 8 & 2 & 5 & 8 \\
\hline \multirow{10}{*}{ I } & 100 & 11.65 & 22.60 & 42.70 & 7.85 & 8.45 & 8.80 & 8.50 & 8.65 & 7.50 & 8.40 & 10.05 & 11.00 \\
\hline & & (17.95) & $(47.20)$ & $(66.10)$ & (5.65) & $(6.30)$ & $(14.70)$ & $(4.50)$ & $(2.10)$ & (3.55) & (6.20) & (3.25) & (3.65) \\
\hline & 250 & 7.45 & 13.15 & 37.75 & 5.30 & 5.30 & 5.25 & 5.50 & 6.25 & 6.10 & 6.60 & 6.80 & 7.90 \\
\hline & & $(7.70)$ & $(28.10)$ & $(74.35)$ & $(1.85)$ & $(1.05)$ & $(3.70)$ & $(1.35)$ & $(0.35)$ & $(0.40)$ & $(2.25)$ & $(0.70)$ & $(0.25)$ \\
\hline & \multirow[t]{2}{*}{500} & 6.15 & 9.05 & 25.90 & 5.75 & 6.25 & 6.00 & 5.40 & 5.65 & 5.30 & 5.10 & 4.75 & 4.95 \\
\hline & & (3.85) & $(15.25)$ & $(59.60)$ & $(1.55)$ & $(0.65)$ & $(1.75)$ & $(1.50)$ & $(0.15)$ & $(0.05)$ & $(1.00)$ & $(0.10)$ & $(0.10)$ \\
\hline & \multirow[t]{2}{*}{1000} & 5.75 & 7.10 & 15.55 & 5.60 & 5.80 & 5.35 & 6.10 & 5.70 & 4.70 & 5.05 & 5.05 & 4.60 \\
\hline & & $(2.55)$ & $(6.55)$ & $(48.25)$ & $(1.70)$ & $(0.25)$ & $(0.40)$ & $(1.10)$ & $(0.10)$ & $(0.05)$ & $(1.15)$ & $(0.05)$ & $(0.00)$ \\
\hline & \multirow[t]{2}{*}{2000} & 5.40 & 6.10 & 11.00 & 5.10 & 4.70 & 4.95 & 5.35 & 5.25 & 5.35 & 5.80 & 6.20 & 5.25 \\
\hline & & $(2.20)$ & $(2.20)$ & $(33.80)$ & $(1.55)$ & $(0.10)$ & $(0.20)$ & $(0.85)$ & $(0.00)$ & $(0.00)$ & $(0.85)$ & $(0.00)$ & $(0.00)$ \\
\hline \multirow{11}{*}{ II } & \multirow{3}{*}{100} & & & & & & & & & & & & \\
\hline & & 12.45 & 23.15 & 40.60 & 8.00 & 8.55 & 9.20 & 8.30 & 9.15 & 9.50 & 11.25 & 10.45 & 11.00 \\
\hline & & $(20.10)$ & $(45.65)$ & $\begin{array}{l}(68.70) \\
\end{array}$ & $(5.90)$ & $(6.55)$ & $(15.80)$ & $(4.15)$ & $(2.20)$ & $(3.30)$ & $(7.35)$ & $(4.00)$ & $(4.50)$ \\
\hline & \multirow[t]{2}{*}{250} & 7.95 & 12.75 & 37.95 & 5.45 & 5.50 & 5.65 & 6.90 & 6.60 & 5.80 & 7.15 & 8.30 & 6.95 \\
\hline & & $(9.30)$ & $(30.25)$ & $(73.95)$ & $(3.70)$ & $(2.05)$ & $(4.35)$ & $(3.55)$ & $(1.10)$ & $(0.70)$ & $(4.80)$ & $(1.30)$ & $(0.55)$ \\
\hline & 500 & 7.05 & 8.55 & 26.25 & 5.10 & 5.15 & 5.00 & 5.65 & 6.25 & 6.10 & 5.55 & 5.35 & 5.75 \\
\hline & & $(5.70)$ & $(16.55)$ & $(61.65)$ & $(3.10)$ & $(1.85)$ & $(1.90)$ & $(3.45)$ & $(0.60)$ & $(0.40)$ & $(4.15)$ & $(0.75)$ & $(0.25)$ \\
\hline & 1000 & 5.85 & 7.20 & 16.00 & 4.80 & 5.45 & 5.55 & 4.30 & 4.95 & 4.75 & 5.05 & 5.65 & 5.85 \\
\hline & & $(4.60)$ & $(9.20)$ & $(49.15)$ & (3.75) & $(0.70)$ & $(1.00)$ & $(3.10)$ & $(0.75)$ & $(0.05)$ & (3.55) & $(0.85)$ & $(0.10)$ \\
\hline & 2000 & 4.85 & 5.55 & 11.15 & 4.35 & 5.30 & 4.75 & 5.50 & 4.55 & 4.30 & 4.90 & 5.65 & 5.95 \\
\hline & & $(4.05)$ & $(4.20)$ & $(34.85)$ & $(3.60)$ & $(1.00)$ & $(0.65)$ & $(2.40)$ & $(0.55)$ & $(0.10)$ & $(2.90)$ & $(0.30)$ & $(0.00)$ \\
\hline & & & & & & & & & & & & & \\
\hline & 100 & 11.75 & 23.45 & 38.50 & 8.30 & 8.15 & 10.30 & 8.55 & 8.95 & 8.55 & 9.40 & 10.95 & 11.45 \\
\hline & & $(21.25)$ & $(48.05)$ & $(71.30)$ & $(6.15)$ & $(6.50)$ & $(15.30)$ & $(5.10)$ & $(1.70)$ & $(2.60)$ & $(6.80)$ & (3.95) & $(3.45)$ \\
\hline & 250 & 8.05 & 12.75 & 35.80 & 5.75 & 6.35 & 6.55 & 6.40 & 5.65 & 5.35 & 6.35 & 6.30 & 6.80 \\
\hline & & $(8.60)$ & $(28.55)$ & $(76.45)$ & $(3.60)$ & $(3.20)$ & $(4.85)$ & $(3.75)$ & $(1.00)$ & $(0.65)$ & $(4.15)$ & $(1.55)$ & $(0.70)$ \\
\hline & 500 & 7.10 & 8.90 & 24.80 & 5.25 & 4.80 & 5.00 & 4.85 & 4.65 & 4.80 & 5.25 & 5.80 & 5.20 \\
\hline III & & $(6.50)$ & $(18.10)$ & $(59.95)$ & $(4.15)$ & $(1.55)$ & $(2.20)$ & $(4.20)$ & $(0.90)$ & $(0.30)$ & $(4.35)$ & $(1.10)$ & $(0.15)$ \\
\hline & 1000 & 5.30 & 6.60 & 15.40 & 6.00 & 5.40 & 5.70 & 5.15 & 5.05 & 5.70 & 5.85 & 6.25 & 5.60 \\
\hline & & $(5.30)$ & $(9.30)$ & $(49.75)$ & $(3.90)$ & $(1.20)$ & $(1.15)$ & (3.75) & $(0.90)$ & $(0.25)$ & $(4.10)$ & $(1.00)$ & $(0.25)$ \\
\hline & 2000 & 5.30 & 5.85 & 12.30 & 5.30 & 5.30 & 4.45 & 5.25 & 5.25 & 5.15 & 5.25 & 5.50 & 6.15 \\
\hline & & $(4.05)$ & $(5.60)$ & $(34.50)$ & $(3.80)$ & $(1.10)$ & $(0.35)$ & $(3.25)$ & $(1.00)$ & $(0.30)$ & $(3.70)$ & $(0.70)$ & $(0.25)$ \\
\hline & & & & & & & & & & & & & \\
\hline & 100 & 13.30 & 22.95 & 40.35 & 7.80 & 8.95 & 8.20 & 7.45 & 9.20 & 7.15 & 10.05 & 10.55 & 10.90 \\
\hline & & $(19.60)$ & $(47.90)$ & $(68.50)$ & $(7.10)$ & $(6.55)$ & $(16.95)$ & $(5.70)$ & $(2.75)$ & $(3.40)$ & $(7.00)$ & $(4.90)$ & $(3.90)$ \\
\hline & 250 & 6.55 & 12.95 & 38.35 & 5.45 & 6.65 & 6.95 & 7.05 & 7.10 & 6.25 & 6.50 & 6.20 & 7.00 \\
\hline & & $(10.75)$ & $(29.55)$ & $(76.20)$ & $(4.85)$ & $(2.55)$ & $(5.35)$ & $(4.80)$ & $(1.45)$ & $(0.55)$ & $(3.95)$ & $(1.30)$ & $(0.65)$ \\
\hline IV & 500 & 6.30 & 8.70 & 23.90 & 4.95 & 5.00 & 4.55 & 5.15 & 6.35 & 6.45 & 6.75 & 5.65 & 6.40 \\
\hline IV & & $(6.75)$ & $(17.45)$ & $(63.45)$ & $(4.50)$ & $(2.10)$ & $(1.90)$ & $(3.55)$ & $(0.65)$ & $(0.35)$ & $(4.40)$ & $(1.60)$ & $(0.50)$ \\
\hline & 1000 & 5.10 & 5.95 & 16.25 & 5.45 & 5.35 & 4.65 & 6.30 & 6.30 & 6.40 & 4.60 & 5.20 & 5.45 \\
\hline & & $(4.45)$ & $(9.35)$ & $(49.20)$ & (3.15) & $(1.40)$ & $(1.35)$ & $(2.70)$ & $(0.55)$ & $(0.15)$ & (3.90) & $(1.40)$ & $(0.05)$ \\
\hline & 2000 & 4.65 & 5.90 & 11.05 & 6.00 & 5.20 & 5.85 & 4.30 & 5.15 & 4.85 & 3.80 & 5.05 & 5.40 \\
\hline & & $(4.10)$ & $(5.05)$ & $(35.20)$ & (3.25) & $(0.95)$ & $(0.45)$ & (3.30) & $(0.85)$ & $(0.35)$ & $(4.00)$ & $(1.25)$ & $(0.25)$ \\
\hline & & & & & & & & & & & & & \\
\hline & 100 & 11.40 & 22.90 & 38.05 & 8.20 & 9.15 & 9.00 & 8.10 & 7.95 & 8.20 & 10.60 & 10.75 & 10.90 \\
\hline & & $(19.55)$ & $(48.05)$ & $\begin{array}{l}(71.20) \\
\end{array}$ & $(6.45)$ & (6.95) & (18.85) & (5.05) & (3.35) & (3.60) & (7.55) & $(4.40)$ & (3.95) \\
\hline & 250 & 7.60 & 12.15 & 37.20 & 5.85 & 5.70 & 6.15 & 6.05 & 6.15 & 5.55 & 5.80 & 7.65 & 7.50 \\
\hline & & $(11.50)$ & $(29.20)$ & $(76.85)$ & $(5.40)$ & $(2.70)$ & $(4.30)$ & (3.70) & $(1.05)$ & $(0.75)$ & $(4.30)$ & $(2.20)$ & $(0.85)$ \\
\hline$V$ & 500 & 6.25 & 7.80 & 26.00 & 5.80 & 6.00 & 4.70 & 5.20 & 5.75 & 5.45 & 5.05 & 6.10 & 5.70 \\
\hline & & $(5.95)$ & $(18.00)$ & $(60.00)$ & $(4.00)$ & $(2.00)$ & $(2.30)$ & $(3.80)$ & $(1.05)$ & $(0.50)$ & $(4.05)$ & $(0.95)$ & $(0.35)$ \\
\hline & 1000 & 6.20 & 6.30 & 17.35 & 5.55 & 5.60 & 5.00 & 5.25 & 5.25 & 5.40 & 4.90 & 5.05 & 4.95 \\
\hline & & $(5.20)$ & $(10.45)$ & $(48.60)$ & $(3.60)$ & $(1.65)$ & $(1.40)$ & $(3.80)$ & $(1.35)$ & $(0.30)$ & $(3.05)$ & $(1.00)$ & $(0.20)$ \\
\hline & 2000 & 5.90 & 5.15 & 10.75 & 4.80 & 5.05 & 5.10 & 5.55 & 5.40 & 5.15 & 5.45 & 4.70 & 4.90 \\
\hline & & $(4.85)$ & $(5.50)$ & $(34.90)$ & $(3.80)$ & $(1.80)$ & $(1.30)$ & (3.35) & $(0.95)$ & $(0.25)$ & $(3.10)$ & $(0.90)$ & $(0.20)$ \\
\hline
\end{tabular}


Table 3: Distribution of BDS (Gaussian Innovations)

\begin{tabular}{|c|c|c|c|c|c|c|c|c|c|c|c|c|c|}
\hline $\begin{array}{c}\text { Case } \\
I\end{array}$ & \multicolumn{3}{|c|}{$\varepsilon / \sigma=1$} & \multicolumn{3}{|c|}{$\varepsilon / \sigma=1.5$} & & \multicolumn{3}{|c|}{$\varepsilon / \sigma=1$} & \multicolumn{3}{|c|}{$\varepsilon / \sigma=1.5$} \\
\hline$T=100$ & $\mathbf{m}=2$ & 5 & 8 & 2 & 5 & 8 & $\mathbf{T}=\mathbf{5 0 0}$ & 2 & 5 & 8 & 2 & 5 & 8 \\
\hline$<-1.96$ & 4.30 & 3.85 & 3.45 & 4.45 & 5.15 & 4.05 & & 2.85 & 3.00 & 2.15 & 2.40 & 2.75 & 2.50 \\
\hline$>1.96$ & 3.55 & 4.60 & 5.35 & 4.05 & 3.50 & 3.45 & & 2.90 & 3.25 & 3.85 & 3.00 & 2.90 & 2.80 \\
\hline Mean & -0.15 & -0.20 & -0.19 & -0.19 & -0.29 & -0.30 & & -0.06 & -0.08 & -0.06 & -0.09 & -0.11 & -0.12 \\
\hline St.Dev. & 1.11 & 1.16 & 1.26 & 1.12 & 1.11 & 1.09 & & 1.02 & 1.03 & 1.05 & 1.03 & 1.02 & 1.02 \\
\hline Skewn. & 0.23 & 0.68 & 1.36 & 0.29 & 0.35 & 0.53 & & 0.19 & 0.19 & 0.52 & 0.28 & 0.27 & 0.29 \\
\hline \multirow[t]{2}{*}{ Kurt. } & 3.09 & 3.75 & 7.88 & 3.13 & 3.01 & 3.23 & & 3.05 & 2.93 & 3.44 & 3.12 & 3.08 & 3.02 \\
\hline & \multicolumn{6}{|c|}{$T=1000$} & & \multicolumn{6}{|c|}{$\mathbf{T}=\mathbf{2 0 0 0}$} \\
\hline$<-1.96$ & 2.60 & 2.75 & 1.85 & 2.50 & 2.50 & 2.15 & & 2.70 & 2.25 & 2.10 & 2.60 & 2.55 & 2.45 \\
\hline$>1.96$ & 3.00 & 3.05 & 3.50 & 3.60 & 3.20 & 2.55 & & 2.40 & 2.45 & 2.85 & 2.75 & 2.70 & 2.90 \\
\hline Mean & -0.03 & -0.06 & -0.06 & -0.01 & -0.05 & -0.06 & & -0.04 & -0.04 & -0.04 & 0.04 & -0.01 & -0.03 \\
\hline St.Dev. & 1.01 & 1.01 & 1.03 & 1.04 & 1.01 & 1.00 & & 1.00 & 1.00 & 1.01 & 1.03 & 1.02 & 1.01 \\
\hline Skewn. & 0.08 & 0.23 & 0.41 & 0.18 & 0.20 & 0.29 & & 0.02 & 0.17 & 0.27 & 0.01 & 0.09 & 0.17 \\
\hline Kurt. & 3.08 & 2.99 & 3.08 & 3.09 & 3.26 & 3.32 & & 2.96 & 3.02 & 3.10 & 2.94 & 3.11 & 3.03 \\
\hline $\begin{array}{c}\text { Case } \\
\text { III }\end{array}$ & \multicolumn{6}{|c|}{$T=100$} & & \multicolumn{6}{|c|}{$T=500$} \\
\hline$<-1.96$ & 4.45 & 4.15 & 4.65 & 4.75 & 5.25 & 4.25 & & 2.40 & 1.85 & 1.45 & 2.55 & 2.15 & 2.05 \\
\hline$>1.96$ & 3.85 & 4.00 & 5.65 & 3.80 & 3.70 & 4.30 & & 2.85 & 2.95 & 3.55 & 2.30 & 2.50 & 2.75 \\
\hline Mean & -0.16 & -0.23 & -0.23 & -0.21 & -0.26 & -0.24 & & -0.07 & -0.11 & -0.11 & -0.08 & -0.11 & -0.12 \\
\hline St.Dev. & 1.13 & 1.17 & 1.23 & 1.13 & 1.13 & 1.14 & & 1.02 & 1.02 & 1.02 & 1.00 & 1.00 & 1.00 \\
\hline Skewn. & 0.31 & 0.59 & 0.91 & 0.29 & 0.34 & 0.52 & & 0.17 & 0.37 & 0.62 & 0.21 & 0.28 & 0.34 \\
\hline \multirow{2}{*}{ Kurt. } & 3.07 & 3.77 & 4.71 & 2.97 & 3.03 & 3.32 & & 2.97 & 3.21 & 3.78 & 2.90 & 3.07 & 3.18 \\
\hline & \multicolumn{6}{|c|}{$\mathrm{T}=1000$} & & \multicolumn{6}{|c|}{$\mathrm{T}=2000$} \\
\hline$<-1.96$ & 2.90 & 2.70 & 1.90 & 2.35 & 2.20 & 2.30 & & 2.75 & 2.70 & 1.65 & 2.60 & 2.40 & 2.60 \\
\hline$>1.96$ & 3.10 & 2.70 & 3.80 & 2.80 & 2.85 & 3.40 & & 2.55 & 2.60 & 2.80 & 2.65 & 2.85 & 2.55 \\
\hline Mean & -0.08 & -0.11 & -0.09 & -0.05 & -0.07 & -0.07 & & -0.03 & -0.05 & -0.05 & -0.02 & -0.04 & -0.05 \\
\hline St.Dev. & 1.02 & 1.02 & 1.03 & 1.00 & 1.01 & 1.02 & & 1.01 & 0.99 & 0.99 & 1.02 & 1.01 & 1.01 \\
\hline Skewn. & 0.16 & 0.29 & 0.51 & 0.18 & 0.21 & 0.31 & & -0.03 & 0.10 & 0.26 & 0.07 & 0.14 & 0.14 \\
\hline Kurt. & 3.22 & 3.18 & 3.34 & 3.02 & 2.96 & 3.05 & & 2.96 & 2.91 & 2.99 & 3.21 & 2.97 & 3.01 \\
\hline $\begin{array}{c}\text { Case } \\
V\end{array}$ & \multicolumn{6}{|c|}{$\mathrm{T}=100$} & & \multicolumn{6}{|c|}{$\mathrm{T}=500$} \\
\hline$<-1.96$ & 4.60 & 4.30 & 3.10 & 4.45 & 4.40 & 4.05 & & 2.60 & 2.60 & 1.05 & 2.85 & 3.10 & 2.70 \\
\hline$>1.96$ & 3.60 & 4.85 & 5.90 & 3.65 & 3.55 & 4.15 & & 3.20 & 3.40 & 3.65 & 2.35 & 2.65 & 2.75 \\
\hline Mean & -0.18 & -0.21 & -0.17 & -0.17 & -0.24 & -0.25 & & -0.05 & -0.07 & -0.08 & -0.11 & -0.13 & -0.13 \\
\hline St.Dev. & 1.13 & 1.17 & 1.27 & 1.13 & 1.14 & 1.14 & & 1.05 & 1.04 & 1.03 & 1.00 & 1.02 & 1.03 \\
\hline Skewn. & 0.30 & 0.61 & 1.29 & 0.36 & 0.47 & 0.67 & & 0.24 & 0.30 & 0.50 & 0.16 & 0.19 & 0.31 \\
\hline \multirow[t]{2}{*}{ Kurt. } & 3.01 & 3.56 & 6.28 & 3.47 & 3.30 & 3.89 & & 2.87 & 3.13 & 3.24 & 2.90 & 2.97 & 3.01 \\
\hline & \multicolumn{6}{|c|}{$T=1000$} & & \multicolumn{6}{|c|}{$\mathrm{T}=\mathbf{2 0 0 0}$} \\
\hline$<-1.96$ & 2.75 & 2.70 & 1.80 & 2.85 & 2.15 & 2.25 & & 2.85 & 1.85 & 1.75 & 2.10 & 2.30 & 2.50 \\
\hline$>1.96$ & 2.80 & 2.90 & 3.20 & 2.40 & 3.10 & 3.15 & & 1.95 & 3.20 & 3.35 & 3.45 & 3.10 & 2.65 \\
\hline Mean & -0.07 & -0.09 & -0.09 & -0.04 & -0.04 & -0.05 & & -0.06 & -0.04 & -0.03 & -0.02 & -0.02 & -0.03 \\
\hline St.Dev. & 1.04 & 1.02 & 1.02 & 1.02 & 1.04 & 1.03 & & 0.99 & 1.00 & 1.00 & 1.05 & 1.00 & 0.99 \\
\hline Skewn. & 0.13 & 0.12 & 0.33 & 0.06 & 0.20 & 0.27 & & 0.05 & 0.19 & 0.29 & 0.15 & 0.17 & 0.17 \\
\hline Kurt. & 3.00 & 2.85 & 3.02 & 3.15 & 3.02 & 3.02 & & 3.03 & 2.94 & 3.01 & 2.89 & 3.04 & 3.02 \\
\hline
\end{tabular}


Table 4: Size of BDS (and BDS ${ }^{*}$ in parentheses) for the t(4)- Distribution

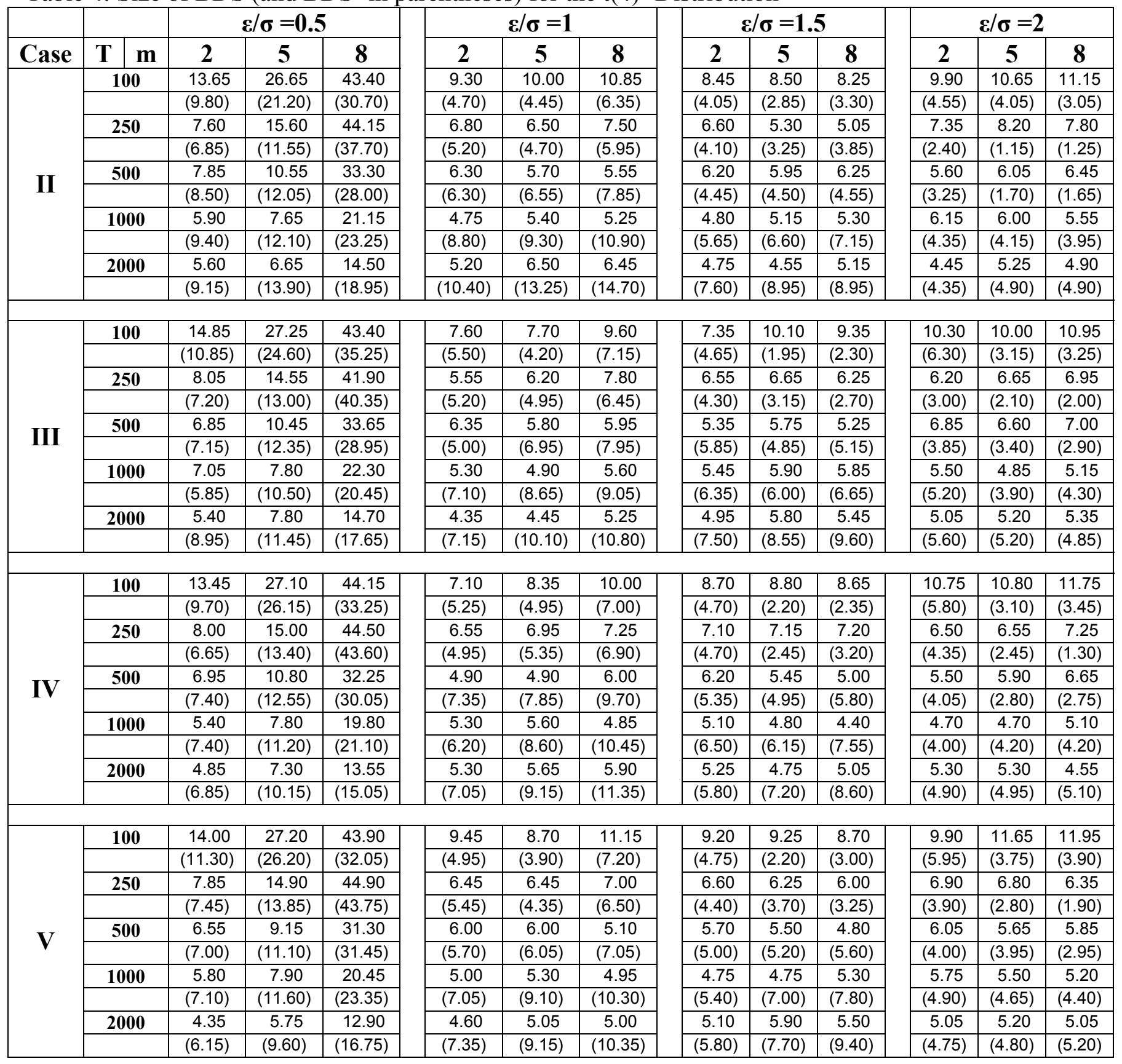


Table 5: Size of BDS (and BDS* in parentheses) for the $\mathrm{X}^{2}(1)$ - Distribution

\begin{tabular}{|c|c|c|c|c|c|c|c|c|c|c|c|c|c|}
\hline \multirow[b]{2}{*}{ Case } & \multirow[b]{2}{*}{$\mathbf{T}$} & \multicolumn{3}{|c|}{$\varepsilon / \sigma=0.5$} & \multicolumn{3}{|c|}{$\varepsilon / \sigma=1$} & \multicolumn{3}{|c|}{$\varepsilon / \sigma=1.5$} & \multicolumn{3}{|c|}{$\varepsilon / \sigma=2$} \\
\hline & & 2 & 5 & 8 & 2 & 5 & 8 & 2 & 5 & 8 & 2 & 5 & 8 \\
\hline \multirow{10}{*}{ I } & 100 & 11.60 & 13.25 & 14.05 & 8.60 & 7.90 & 8.30 & 7.80 & 7.75 & 7.70 & 8.85 & 10.60 & 10.95 \\
\hline & & $(7.10)$ & $(7.60)$ & $(9.80)$ & $(6.00)$ & $(4.60)$ & $(5.80)$ & (5.20) & $(3.00)$ & $(3.05)$ & $(5.30)$ & $(4.15)$ & (3.70) \\
\hline & 250 & 9.25 & 9.65 & 11.15 & 6.60 & 6.50 & 6.35 & 5.35 & 5.40 & 6.15 & 5.35 & 6.60 & 5.85 \\
\hline & & $(5.70)$ & $(6.40)$ & $(8.60)$ & (4.35) & (3.65) & $(4.60)$ & $(3.60)$ & (2.05) & $(2.20)$ & (3.10) & (1.45) & $(1.75)$ \\
\hline & 500 & 7.45 & 8.50 & 10.95 & 6.45 & 5.60 & 5.20 & 5.20 & 5.40 & 5.70 & 5.65 & 6.30 & 6.40 \\
\hline & & (5.95) & $(8.00)$ & (8.35) & $(4.00)$ & $(5.30)$ & $(5.70)$ & $(3.30)$ & $(2.60)$ & $(2.85)$ & (2.85) & (1.35) & $(1.35)$ \\
\hline & 1000 & 5.55 & 5.50 & 6.95 & 4.95 & 5.15 & 6.00 & 4.75 & 4.65 & 5.15 & 5.45 & 5.20 & 5.60 \\
\hline & & (5.65) & $(5.80)$ & (7.55) & $(4.80)$ & $(4.85)$ & $(4.60)$ & (3.75) & $(3.45)$ & (3.55) & $(2.55)$ & $(1.30)$ & $(1.50)$ \\
\hline & 2000 & 5.35 & 5.45 & 6.80 & 5.45 & 5.00 & 5.15 & 5.45 & 5.20 & 5.55 & 4.65 & 5.15 & 5.30 \\
\hline & & (6.15) & $(6.40)$ & (6.30) & (5.25) & $(4.90)$ & (4.85) & (3.90) & (3.95) & (4.55) & (2.95) & (1.65) & (1.45) \\
\hline \multirow{11}{*}{ II } & & & & & & & & & & & & & \\
\hline & 100 & 12.05 & 13.85 & 15.40 & 9.20 & 8.10 & 8.25 & 7.40 & 8.45 & 7.95 & 9.35 & 10.00 & 11.40 \\
\hline & & $(8.25)$ & $(9.20)$ & $(12.40)$ & $(5.70)$ & $(5.30)$ & (5.95) & $(5.15)$ & (3.45) & $(4.25)$ & $(5.20)$ & (3.35) & (3.70) \\
\hline & 250 & 8.35 & 9.45 & 11.75 & 7.50 & 7.35 & 7.15 & 6.70 & 6.40 & 6.65 & 6.30 & 6.95 & 7.05 \\
\hline & & $(5.65)$ & $(6.55)$ & $(9.65)$ & $(4.80)$ & $(4.30)$ & $(5.20)$ & $(4.45)$ & (3.30) & $(2.75)$ & (3.10) & (2.10) & (1.85) \\
\hline & 500 & 7.15 & 7.25 & 9.55 & 6.30 & 6.25 & 5.85 & 6.20 & 5.80 & 5.60 & 6.15 & 6.15 & 6.15 \\
\hline & & $(6.50)$ & (7.95) & $(8.60)$ & $(5.80)$ & $(4.15)$ & $(4.10)$ & $(3.35)$ & $(3.05)$ & $(3.25)$ & (3.20) & $(2.15)$ & $(1.90)$ \\
\hline & 1000 & 6.75 & 5.40 & 7.90 & 5.70 & 5.65 & 5.30 & 6.45 & 5.65 & 4.70 & 5.80 & 6.15 & 5.95 \\
\hline & & (6.10) & (6.85) & (7.60) & $(5.00)$ & (4.95) & (5.55) & (3.45) & (3.60) & $(2.80)$ & $(2.90)$ & (2.15) & $(2.00)$ \\
\hline & 2000 & 5.75 & 5.10 & 6.15 & 4.65 & 5.00 & 4.90 & 5.10 & 5.10 & 4.90 & 5.45 & 5.25 & 4.85 \\
\hline & & (5.35) & (5.95) & $(6.50)$ & (5.25) & $(5.10)$ & (5.25) & $(4.05)$ & (3.75) & $(3.40)$ & (3.90) & $(2.45)$ & $(2.25)$ \\
\hline & & & & & & & & & & & & & \\
\hline \multirow{10}{*}{ III } & 100 & 11.60 & 14.15 & 16.50 & 8.55 & 7.95 & 8.55 & 8.00 & 7.80 & 7.85 & 9.85 & 9.95 & 10.55 \\
\hline & & $(7.90)$ & $(11.40)$ & $(14.00)$ & $(6.20)$ & $(4.45)$ & $(6.00)$ & $(5.30)$ & $(4.00)$ & $(4.35)$ & $(5.30)$ & (3.70) & $(3.20)$ \\
\hline & 250 & 9.55 & 10.75 & 13.65 & 6.75 & 6.15 & 6.00 & 6.55 & 6.00 & 6.70 & 6.60 & 6.75 & 6.00 \\
\hline & & (6.55) & $(7.40)$ & $(10.30)$ & $(5.90)$ & $(5.40)$ & $(5.70)$ & $(3.80)$ & $(3.10)$ & $(3.20)$ & (3.35) & $(2.50)$ & $(1.45)$ \\
\hline & 500 & 7.55 & 8.65 & 10.85 & 4.85 & 5.35 & 5.70 & 5.00 & 6.05 & 5.95 & 5.60 & 5.85 & 6.00 \\
\hline & & $(5.30)$ & (7.55) & $(8.85)$ & (5.15) & $(4.50)$ & $(4.60)$ & $(5.10)$ & $(3.00)$ & (2.95) & (3.45) & $(2.80)$ & $(2.20)$ \\
\hline & 1000 & 5.85 & 5.15 & 6.30 & 5.35 & 5.35 & 5.60 & 4.75 & 5.95 & 5.10 & 4.35 & 5.20 & 5.20 \\
\hline & & $(6.50)$ & $(5.40)$ & $(6.50)$ & $(7.00)$ & $(6.15)$ & $(5.85)$ & $(4.45)$ & (3.65) & $(3.45)$ & (3.90) & $(3.10)$ & $(2.25)$ \\
\hline & 2000 & 5.25 & 5.65 & 5.75 & 4.60 & 4.60 & 4.00 & 4.90 & 5.10 & 4.95 & 5.35 & 6.00 & 5.35 \\
\hline & & $(5.90)$ & $(6.00)$ & $(7.00)$ & $(5.50)$ & $(5.45)$ & $(5.70)$ & (3.65) & (3.30) & (3.35) & $(4.10)$ & (3.55) & $(2.35)$ \\
\hline \multirow{11}{*}{ IV } & & & & & & & & & & & & & \\
\hline & 100 & 11.65 & 13.80 & 17.10 & 8.80 & 8.00 & 8.35 & 7.95 & 8.55 & 8.95 & 8.55 & 9.30 & 9.70 \\
\hline & & $(8.85)$ & $(8.95)$ & $(12.25)$ & $(6.20)$ & (4.75) & (5.35) & $(5.60)$ & (3.30) & $(3.80)$ & $(6.10)$ & $(4.25)$ & (3.55) \\
\hline & 250 & 8.90 & 12.05 & 14.30 & 5.75 & 5.70 & 6.60 & 5.25 & 5.45 & 5.55 & 5.85 & 6.40 & 6.70 \\
\hline & & (6.25) & (7.95) & (10.45) & $(4.15)$ & $(3.90)$ & $(4.55)$ & $(4.20)$ & (3.45) & $(2.50)$ & (3.65) & $(2.40)$ & (2.35) \\
\hline & 500 & 7.90 & 9.30 & 11.30 & 6.05 & 6.10 & 6.10 & 5.60 & 5.25 & 5.05 & 5.10 & 5.90 & 5.60 \\
\hline & & $(5.85)$ & $(6.40)$ & $(8.90)$ & $(4.55)$ & $(4.75)$ & $(4.55)$ & $(5.35)$ & (3.85) & (3.70) & $(4.05)$ & $(4.00)$ & $(3.40)$ \\
\hline & 1000 & 6.35 & 7.15 & 7.60 & 5.10 & 5.10 & 4.90 & 4.80 & 5.30 & 4.90 & 5.45 & 4.85 & 4.75 \\
\hline & & $(5.35)$ & $(6.30)$ & $(7.10)$ & (5.05) & $(4.45)$ & (5.45) & $(4.95)$ & $(4.15)$ & $(4.30)$ & $(4.05)$ & $(2.85)$ & (2.05) \\
\hline & 2000 & 5.55 & 4.90 & 5.80 & 5.20 & 5.00 & 5.15 & 4.75 & 5.30 & 5.75 & 5.20 & 6.05 & 6.15 \\
\hline & & $(5.40)$ & $(5.55)$ & $(6.10)$ & $(5.10)$ & (5.65) & $(5.85)$ & $(3.70)$ & $(4.50)$ & $(4.05)$ & (3.95) & (3.25) & (2.45) \\
\hline & & & & & & & & & & & & & \\
\hline \multirow{10}{*}{ V } & 100 & 10.45 & 12.75 & 16.25 & 9.70 & 8.45 & 8.50 & 7.30 & 8.75 & 8.90 & 8.85 & 9.70 & 10.75 \\
\hline & & $(7.10)$ & $(9.60)$ & $(13.35)$ & (6.70) & $(5.20)$ & $(6.60)$ & $(4.70)$ & $(3.50)$ & $(4.55)$ & (6.50) & (3.45) & (3.50) \\
\hline & 250 & 8.05 & 10.80 & 14.10 & 5.65 & 6.15 & 5.80 & 5.50 & 7.20 & 6.70 & 5.90 & 7.05 & 7.10 \\
\hline & & $(6.05)$ & $(8.25)$ & $(11.20)$ & $(4.75)$ & $(4.10)$ & $(4.10)$ & $(4.10)$ & (3.35) & $(2.75)$ & $(3.80)$ & $(3.45)$ & $(2.15)$ \\
\hline & 500 & 7.65 & 8.85 & 10.40 & 5.70 & 5.40 & 5.10 & 5.10 & 4.95 & 5.10 & 6.45 & 6.90 & 6.30 \\
\hline & & $(6.80)$ & (6.65) & $(8.90)$ & $(5.10)$ & $(5.50)$ & (6.35) & $(5.10)$ & $(4.05)$ & $(3.40)$ & $(4.00)$ & (3.85) & $(3.10)$ \\
\hline & 1000 & 6.60 & 7.90 & 8.20 & 6.00 & 6.05 & 5.05 & 5.30 & 5.15 & 5.75 & 4.55 & 5.00 & 5.85 \\
\hline & & (5.60) & $(4.80)$ & (6.15) & (6.65) & $(5.70)$ & $(5.40)$ & $(5.30)$ & (5.25) & $(4.85)$ & (4.75) & (3.90) & $(2.80)$ \\
\hline & 2000 & 6.15 & 5.55 & 6.00 & 5.30 & 4.95 & 4.35 & 5.20 & 5.35 & 4.70 & 4.35 & 5.60 & 5.50 \\
\hline & & $(4.60)$ & (6.65) & (6.95) & $(4.90)$ & $(4.20)$ & $(4.40)$ & $(4.60)$ & (3.55) & (3.75) & $(4.15)$ & (3.65) & $(2.90)$ \\
\hline
\end{tabular}


Figure 1: Regions for the $\mathrm{N}(0,1)$ distribution

Z N $(\mathbf{0 , 1 )}$

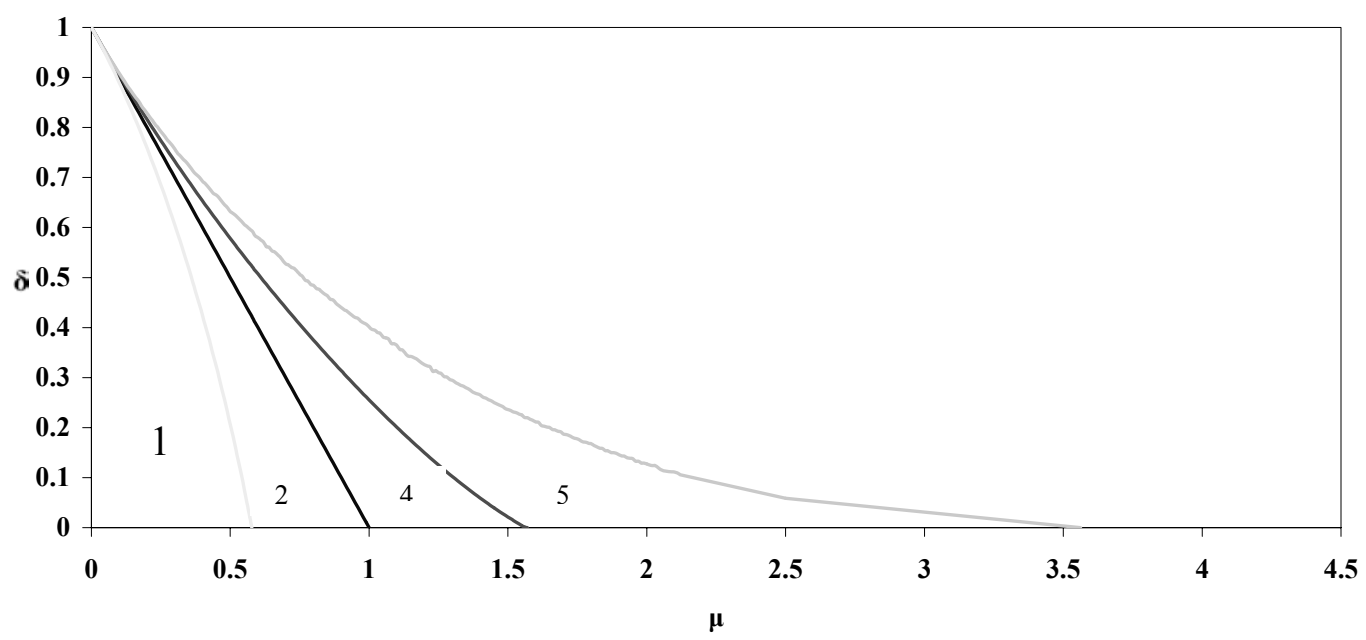

Figure 2: Distribution of BDS* (Gaussian Innovations, Region 1, $\varepsilon / \sigma=1, \mathrm{~m}=2$ )

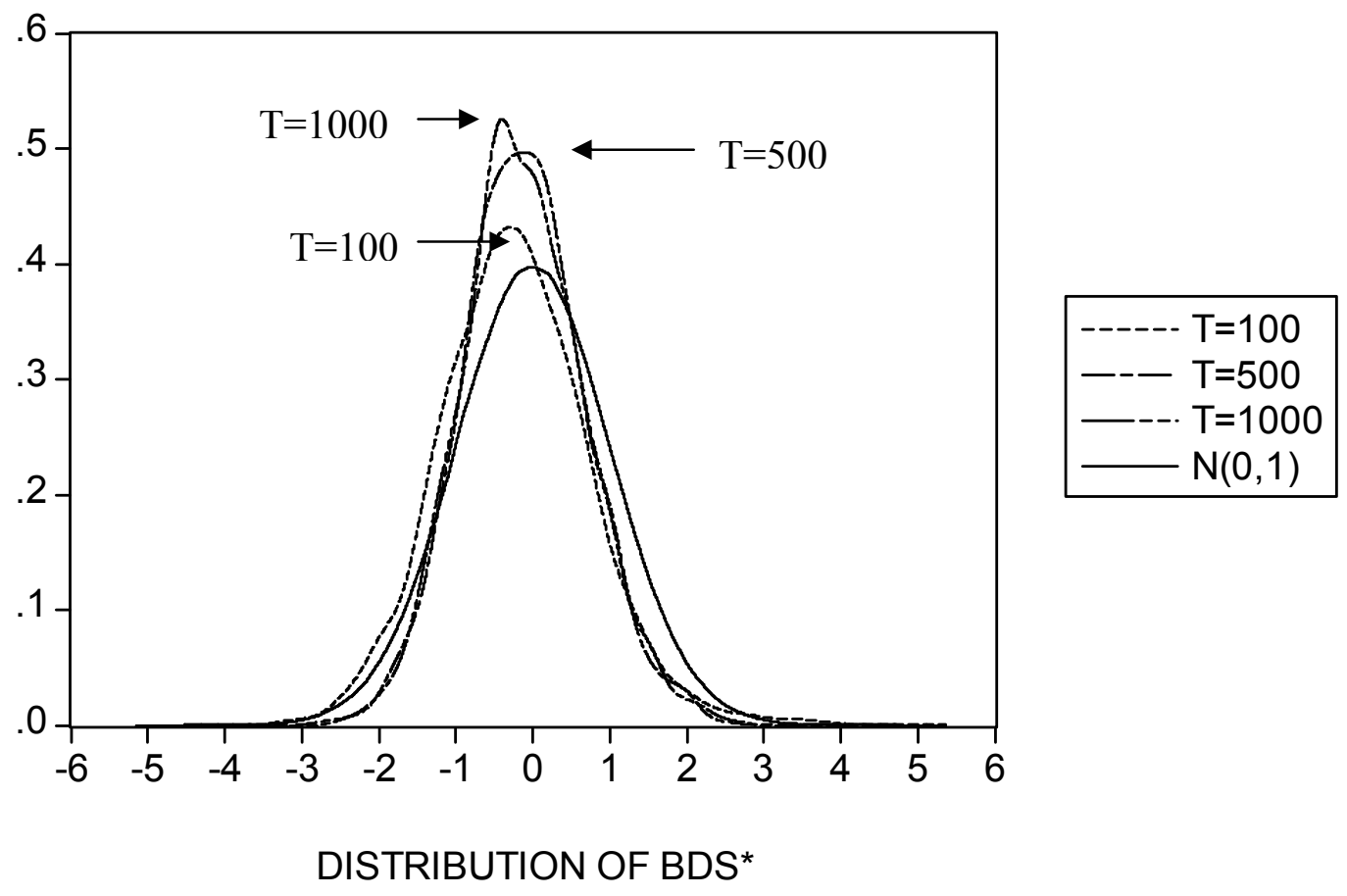


Figure 3: Distribution of BDS (Gaussian Innovations, Region $1, \varepsilon / \sigma=1, \mathrm{~m}=2$ )

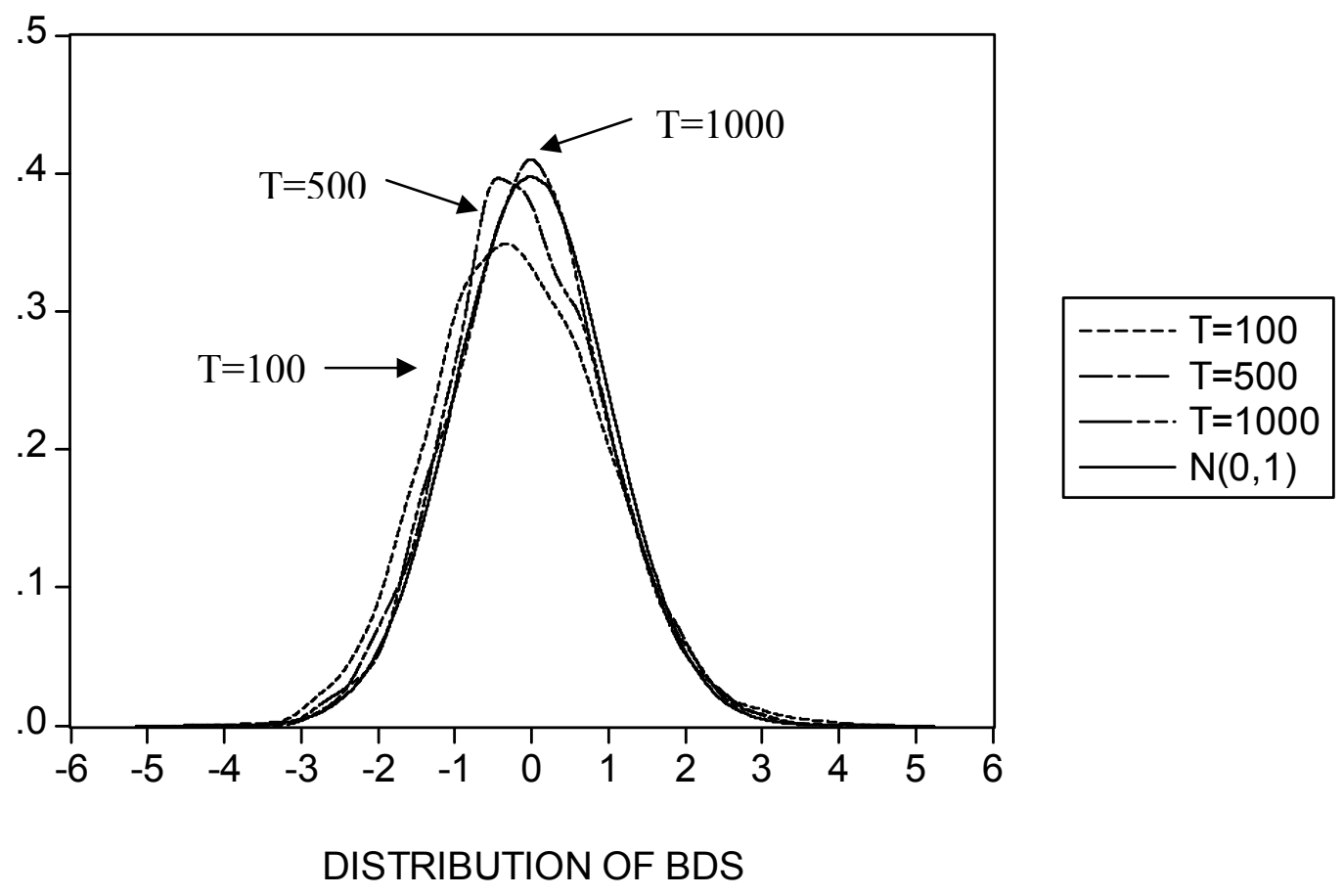

Figure 4: Distribution of BDS (Gaussian Innovations, $\mathrm{T}=1000, \varepsilon / \sigma=1, \mathrm{~m}=2$ )

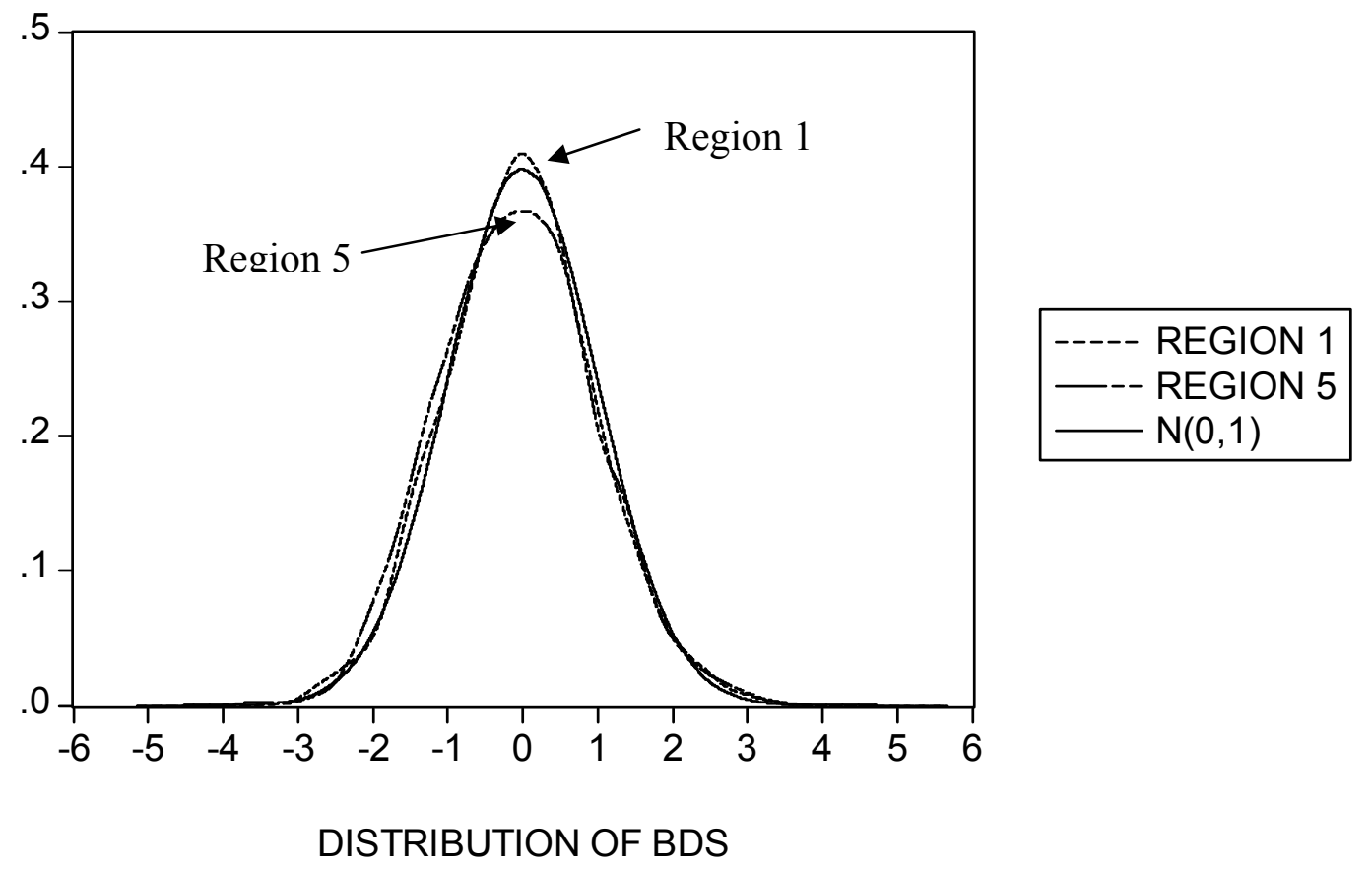


Figure 5: Regions for the $\mathrm{t}(4)$-distribution

$$
\text { Z t-stud(4) }
$$

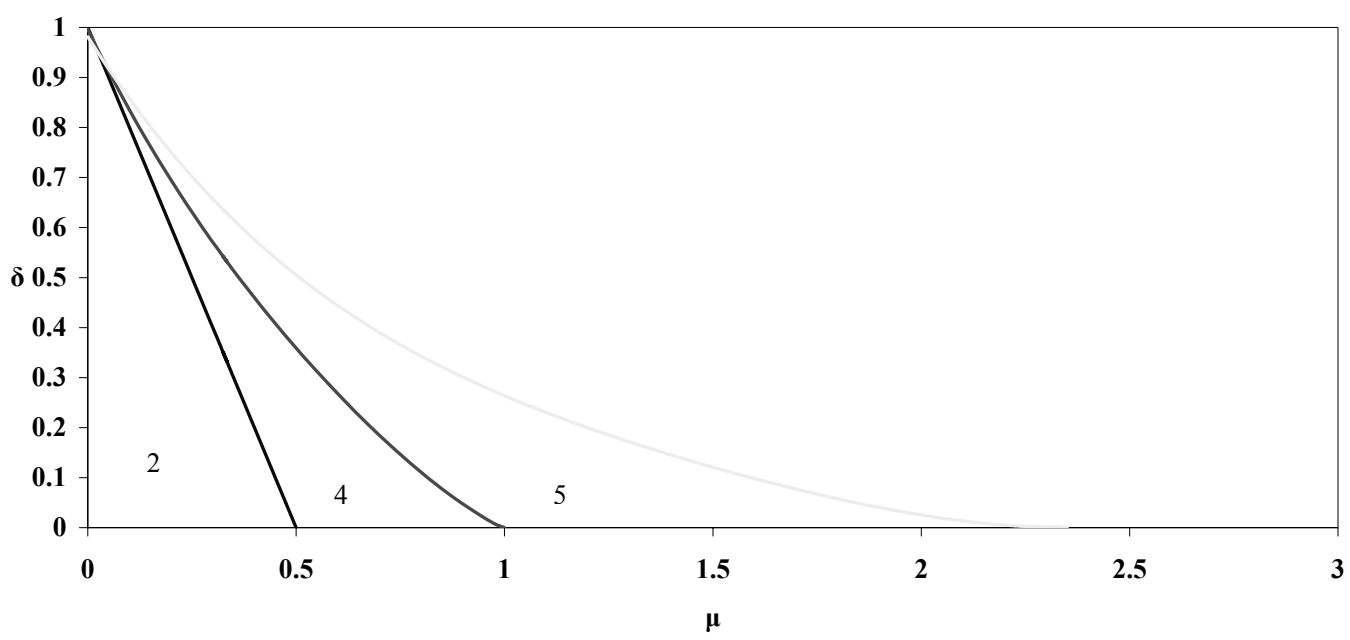

Figure 6: Distribution of $\operatorname{BDS}^{*}(\mathrm{t}(4)$-Distribution, Region $1, \varepsilon / \sigma=1, \mathrm{~m}=2$ )

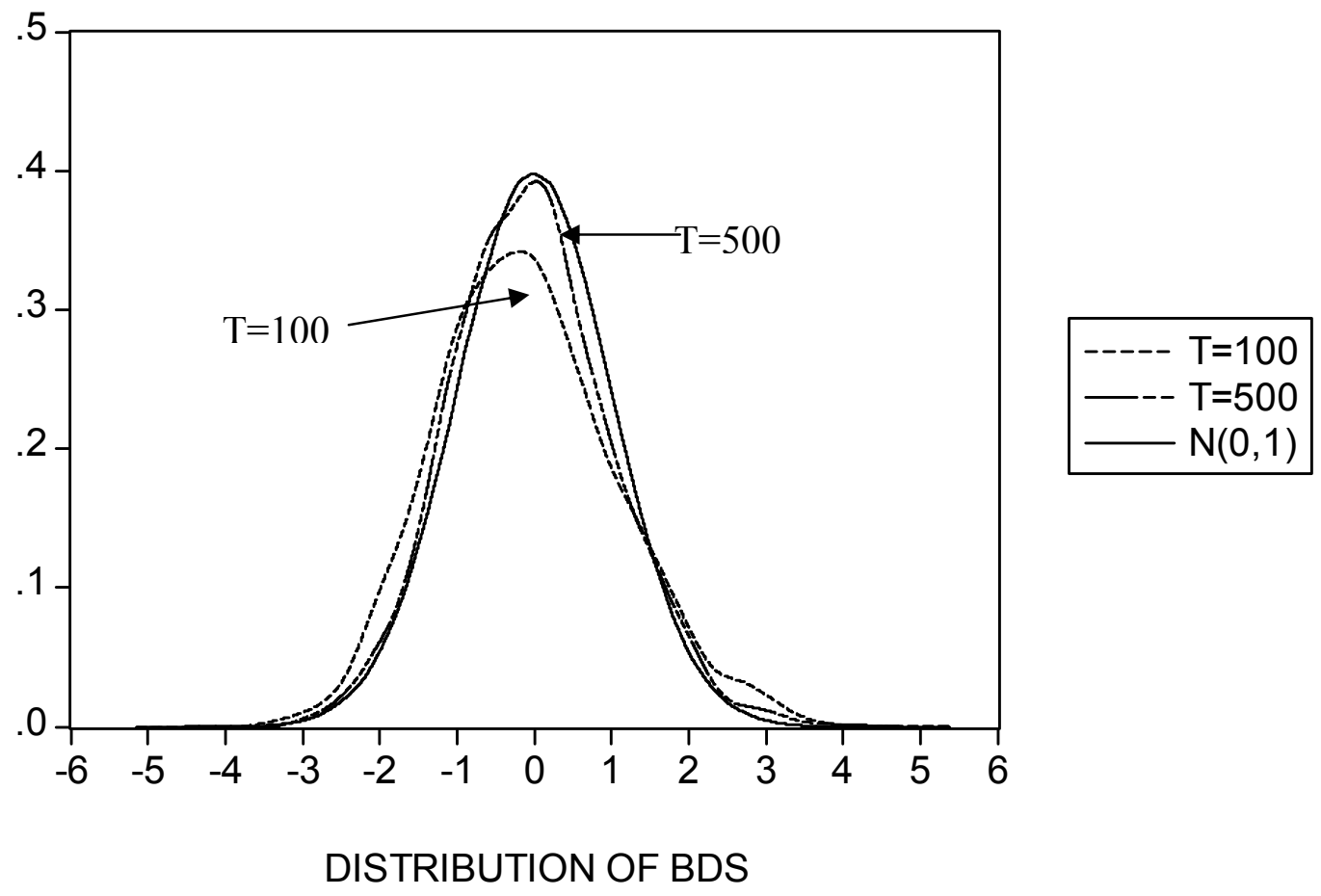


Figure 7: Regions for the $\chi^{2}(1)$-distribution

$$
\mathrm{Z} \sim \chi^{2}(\mathbf{1})
$$

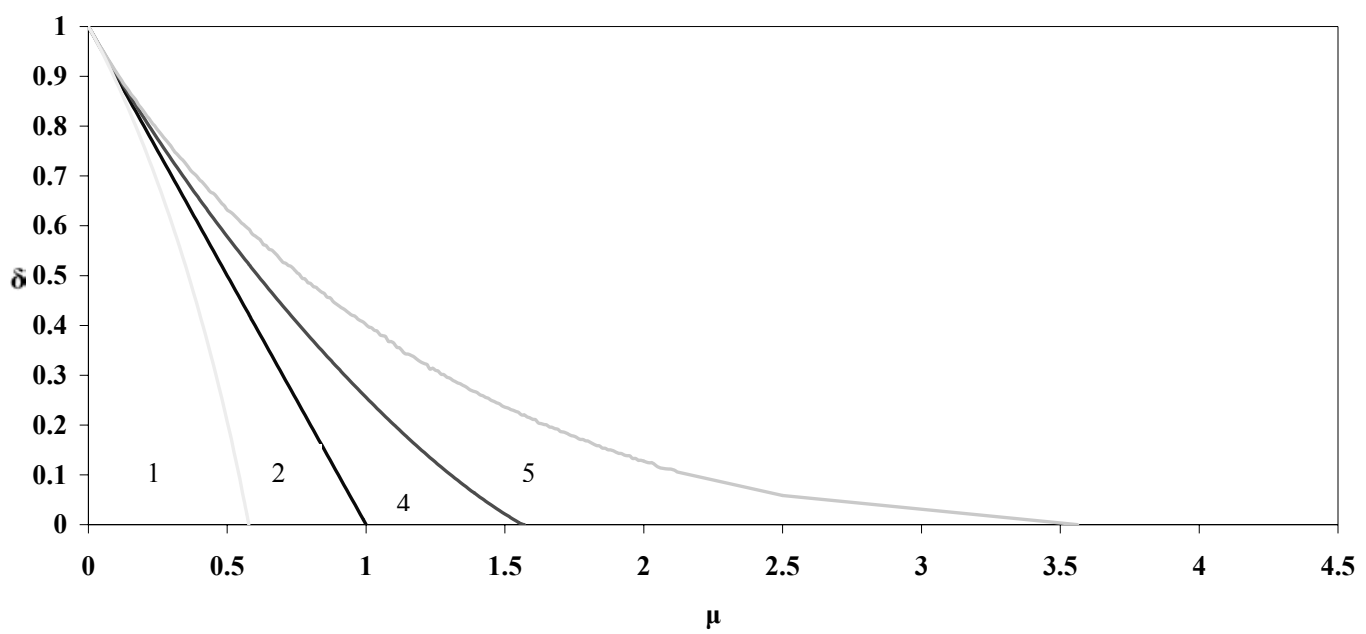

Figure 8: Percentage Mean Absolute Bias of the QML Estimator of $\mu$ (Region 2)

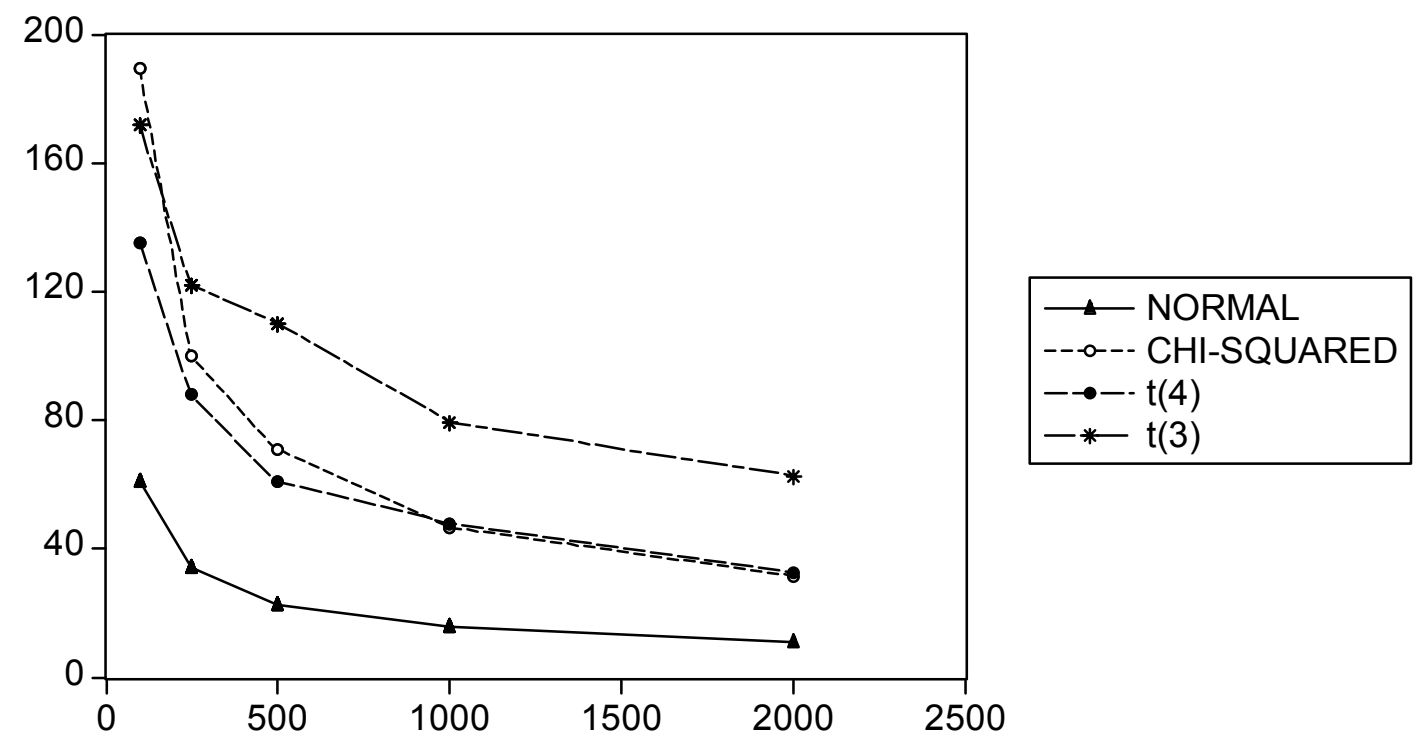

SAMPLE SIZE 